\title{
Traditional Chinese-Hong Kong version of Forgotten Joint Score-12 (FJS-12) for patients with osteoarthritis of the knee underwent joint replacement surgery: cross-cultural and sub-cultural adaptation, and validation
}

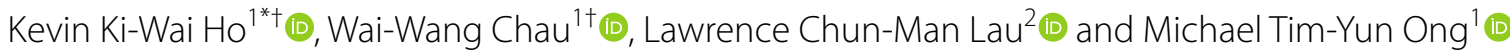

\begin{abstract}
Background: A patient-reported outcome (PRO) tool which reflects the outcomes of patients underwent total knee arthroplasty (TKA) are important to be "ceiling effect free" which commonly used PRO tools face. Forgotten joint score-12 (FJS-12) has been proved to reduce or even free from ceiling effect. FJS-12 has been translated to different languages. The objectives of this study are to validate FJS-12 in Traditional Chinese-Hong Kong language and look for the goodness of FJS-12 still exist in this language adapted FJS-12 version.

Methods: FJS-12 was administered to 75 patients whose majority was obese underwent TKA between September 2019 and March 2020. Patients completed 3 sets of questionnaires (FJS-12, Oxford Knee Score (OKS), and Numeric Rating Scale (NRS)) twice, 2 weeks apart. Reliability, internal consistency, responsiveness, test-retest agreement and discriminant validity were evaluated.

Results: Reliability of FJS-12 showed moderate to excellent internal consistency (Cronbach's $a=0.870)$. Test-retest reliability of FJS-12 was good (ICC $=0.769$ ). Bland-Altman plot showed good test-retest agreement. Construct validity in terms of correlations between FJS-12 and OKS, and FJS-12 and NRS were moderate at baseline (Pearson's coefficient $r=0.598)$ and good at follow-up $(r=0.879)$. Smallest detectable change (Responsiveness) was higher than MIC. Floor effect was none observed, and ceiling effect was low. Discriminant validity was found to have no significance. BMI (obesity) did not affect FJS-12 outcomes.

Conclusions: The Traditional Chinese-Hong Kong version of FJS-12 showed good test-retest reliability, validity, responsiveness, BMI non-specific, with no floor and low ceiling effects for patients who underwent TKA. Sub-culture differences in individual PRO tools should be considered in certain ethnicities and languages.
\end{abstract}

Keywords: FJS-12, Total knee arthroplasty, Traditional Chinese-Hong Kong, Validation, Sub-cultural adaptation

\section{${ }^{*}$ Correspondence:}

${ }^{\dagger}$ Kevin Ki-Wai Ho and Wai-Wang Chau contributed equally to this work.

${ }^{1}$ Department of Orthopaedics and Traumatology, Chinese University of Hong Kong, Hong Kong SAR, China

Full list of author information is available at the end of the article

\section{Introduction}

Using patient-reported outcome (PRO) aiming at measuring the health-related quality of life (HRQOL) of end stage knee arthritis patients underwent knee arthroplasty has been well received [1]. The use of PRO is proven useful to reflect and understand the HRQOL of the patients original author(s) and the source, provide a link to the Creative Commons licence, and indicate if changes were made. The images or other third party material in this article are included in the article's Creative Commons licence, unless indicated otherwise in a credit line to the material. If material is not included in the article's Creative Commons licence and your intended use is not permitted by statutory regulation or exceeds the permitted use, you will need to obtain permission directly from the copyright holder. To view a copy of this licence, visit http://creativecommons.org/licenses/by/4.0/. The Creative Commons Public Domain Dedication waiver (http://creativeco mmons.org/publicdomain/zero/1.0/) applies to the data made available in this article, unless otherwise stated in a credit line to the data. 
suffering from their disorder severity [1]. PRO also provides timely and appropriate therapeutic and rehabilitation strategies. The success of a disease-specific PRO always comes with their well cross-cultural adaptation capability which make them locality and language friendly [2].

Forgotten Joint Score-12 (FJS-12) is a newly developed well-recognized joint-specific patient-reported outcome (PRO) focusing on patients' awareness of a specific joint in everyday life [3]. Joint awareness is always 'forgotten' until strong sensations come e.g. pain, mild stiffness, subjective dysfunction, or any discomfort [3]. FJS-12 has been introduced in different joint related studies [4-10] together with some "gold standards", such as Western Ontario and McMaster Universities Osteoarthritis Index (WOMAC) [11, 12], Oxford Knee Score (OKS) [13], Knee Injury and Osteoarthritis Outcome Score (KOOS) [14], Knee Society Score (KSS) and Function Score (KFS) [15]. Recent technology allows patients to look for the information concerning their disease symptoms, treatments receiving and expected outcomes. Gaining knowledge benefits the patients and at the same time, they expect better health outcomes as medical technology (knee arthroplasty) advances. Some of the tools mentioned before, as the PRO's internal construct has been developed for years, find themselves difficult to differentiate between higher levels of function and patient satisfaction (i.e. known ceiling and floor effects) nowadays [16]. One of the advantages of FJS-12 is that it has low ceiling and floor effects [3, 17]. FJS-12 is also found to be the most responsive tool comparing with the PRO mentioned above in patients following total knee arthroplasty (TKA) [18]. FJS-12 is developed to assess the outcomes of hip and knee arthroplasty by evaluating a patient's awareness of the artificial joint during twelve activities of daily living. FJS-12 is based upon the assumption that the goal of total knee arthroplasty is a joint patient can "forget" about. Studies started using FJS-12 as the sole PRO assessment tool $[19,20]$ to access knee functions and used to assess the long-term results after TKA [21].

FJS-12 constructs for shoulder, knee and hip joints and the respective questionnaire names following the joint types-FJS-12 Shoulder, FJS-12 Knee, and FJS-12 Hip. The original version of FJS-12 shows good reliability and validity $[3,22,23]$. Different language adapted versions of FJS-12 are available, including Chinese (China), Chinese (Hong Kong), and Chinese (Taiwan) versions.

World Health Organization (WHO) developed a universal measuring tool of the quality of life (QOL) called the WHOQOL Questionnaire, and WHOQOL had been translated to different languages, including Chinese (China), Chinese (Hong Kong), and Chinese
(Taiwan). The development process teams of WHOQOL from mainland China, Hong Kong and Taiwan looked for the similarities and differences among these 3 language versions [24]. The authors found that, although "Chinese" language in the three regions used a similar written and spoken language and was deeply influenced by the same ancient Chinese philosophies, variations still found. The report mentioned that the differences could be attributed to a combination of historical and geo-political factors [24]. Similarities and dissimilarities can be found within subcultures [24]. The similarities and dissimilarities can also be found in other well recognized QOL measures e.g., Short Form36 (SF-36) (SF-36 has China, Hong Kong, and Taiwan versions). Another example of sub-culture difference is also referred to the development of WHOQOL, of which WHOQOL developed USA (American English), Canadian (Canadian English), UK (British English), and Australia (Australian English) versions. That also reflects subcultural differences exist among English speaking countries.

Why is FJS-12 necessary to have the "Traditional ChineseHong Kong" version when "Simplified Chinese-Mandarin Chinese" version and "Traditional Chinese-Taiwan" version are available? To recall, FJS-12 has already been translated to Simplified Chinese-Mandarin Chinese [25], and translated and linguistically validated to Traditional Chinese-Taiwan [26]. "Simplified Chinese" is officially used in mainland China, Singapore and the Chinese community in Malaysia and "Traditional Chinese" is officially and commonly used in Taiwan, Hong Kong, and Macau. In the "Traditional Chinese" societies, however, a fundamental cross-cultural difference between Taiwan and Hong Kong/Macau was reported. In a cross-society comparison of general happiness and personal life satisfaction between 1222 participants from Taiwan and 1044 participants from Hong Kong using an identical survey platform, Hong Kong participants indicated a happier attitude regarding to their recent life than the Taiwanese participants [27]. However, the Taiwanese respondents were more satisfied with their personal quality of life than the Hong Kong respondents. As a result, a Traditional Chinese-Hong Kong version of FJS-12 is necessary to develop although another two Chinese versions is available now.

The purpose of this study is to validate the psychometric properties of FJS-12 by testing the reliability, validity, and responsiveness of the validated FJS-12. Floor and ceiling effects of the translated version were discussed. Oxford Knee Score (OKS) and Numeric Rating Scale (NRS) were conducted in line with the Traditional Chinese-Hong Kong version of FJS-12 and correlations between OKS and FJS-12, and between OKS and NRS were sorted. 


\section{Methods}

Between September 2019 and March 2020, 75 patients who underwent unilateral total knee arthroplasty (TKA) at their end stage of knee osteoarthritis were invited to join this study. The inclusion criteria were 1) male and female patients of any age, 2) presence of unilateral knee osteoarthritis (Kellgren Lawrence scale of III-IV), 3) patients received unilateral total knee arthroplasty at least 1 year before this study, and 4) fluent in Chinese Cantonese reading and comprehension. The exclusion criteria were 1) patients with impaired cognitive function, 2) unable to understand Chinese Cantonese, and 3) unable to self-administer both questionnaires. Informed consent was signed by every participant. Ethics approval was received from the institutional ethics review committee (ethics approval number: 2019.337). The study was performed in accordance with the Declaration of Helsinki and ICH-GCP.

\section{Translation and cross-cultural adaptation}

The translation of the FJS-12 into Traditional ChineseHong Kong version was carried out using "translation and back-translation" method, in accordance with the International Quality of Life Assessment (IQOLA) guideline $[28,29]$. Following the guideline, the FJS-12 was translated from English to Traditional Chinese-Hong Kong by two independent bilingual medical professionals and one non-health worker. The translated version was then back-translated to English by two different independent bilingual medical professionals and another non-health worker. The final version was reviewed and discussed for consistency by all 6 members and subsequently verified (Version 1.1, Appendix 1). Minor modifications were made in different questions for cultural adaptation. The "modifications" were summarized in Appendix 2. "Modifications" concerned about the wordings on the same activities and actions used in different regions, and the changes were meant not to alter the meaning of the questions.

\section{Forgotten Joint Score-12 (FJS-12)}

FJS-12 comprises 12 questions under a 5-point Likert scale (Score $=1$ (never, leftmost) to 5 (mostly, rightmost)). The final score is transformed to a 0-100 scale and then reversed to obtain the final score. Higher score indicates better outcome. Scoring FJS-12 final score follows the recommended scoring algorithm.

\section{Oxford Knee Score (OKS)}

OKS has a similar scoring algorithm with FJS-12. OKS consists of 12 assessment questions concerning pain and function after TKA scoring from 0 to 4 ( 0 being the worst effect and 4 being the best) [13, 30]. Summing up all 12 scores forms the final score, of which the final score ranges from 0 (most severe symptoms) to 48 (least symptoms). In recent cross-cultural adaptation and translation studies on OKS, different translated languages showed good reliability, validity and responsiveness e.g. Arabic [31], Slovenian [32], and Malaysian Chinese, Hong Kong Chinese and Singaporean Chinese [33]. The Hong Kong Traditional Chinese version of OKS was used in this study.

\section{Numeric Rating Scale (NRS)}

NRS has been routinely applied to let the patients rate the pain level on a defined scale. NRS is a single 11 -point numeric scale ranging between 0 and 10, with 0 representing "no pain" and 10 representing the pain extreme [34].

\section{Data collection}

Validated FJS-12 and OKS was administered to the patients during their routine clinic follow-up visits (baseline). NRS was routinely recorded at each patient visit. All patients were invited to come back to the clinic 1-2 weeks after to complete these questionnaires again (follow-up).

Patients' baseline demographics e.g., age, sex, body height, body weight, and side of surgery were collected from electronic medical records from the hospital. Details on education level of patients were not routinely collected, however, obesity in terms of body mass index (BMI) was found to be inversely associated with education level [35].

\section{Statistical analysis}

Demographic characteristics were summarized by mean \pm standard deviation (SD) for numeric data and $\mathrm{N}(\%)$ for categorical data respectively. Reliability was measured through test-retest reliability expressed in terms of intra-class correlation (ICC) (two-way random single measure), internal consistency using Cronbach's Alpha, and smallest detectable change (SDC) [36]. SDC was calculated using the formula: $\mathrm{SDC}=\mathrm{SEM} \times 1.96 \times \sqrt{2}$, where SEM (standard error of mean) $=$ SD [37]. Bland-Altman plot was used to look for test-retest agreement. Correlations between FJS-12 and OKS, and between FJS-12 and NRS were tested to look for the validity between the translated version to a gold standard (construct validity). Responsiveness measuring the measurement error in longitudinal validity under repeated measures was calculated by comparing SDC with minimal important change (MIC). Floor and ceiling effects defined as the percentages of participants scoring the leftmost option "never" ("Floor"; score $=1$ ) and rightmost option "mostly" ("Ceiling"; score=5) in individual 
Table 1 Baseline demographics of the 75 patients underwent total knee arthroplasty

\begin{tabular}{ll}
\hline Baseline demographics & Mean \pm SD (Range) or N(\%) \\
\hline Body height $(\mathrm{m})$ & $1.56 \pm 0.08(1.37,1.76)$ \\
Body weight $(\mathrm{kg})$ & $66.70 \pm 10.31(51.0,93.3)$ \\
$\mathrm{BMI}\left(\mathrm{kg} / \mathrm{m}^{2}\right)$ & $27.48 \pm 4.22(19.66,37.24)$ \\
$\mathrm{BMI}$ & \\
$\quad$ Normal & $12(16.0)$ \\
$\quad$ Overweight & $7(9.4)$ \\
$\quad$ Obese & $56(74.6)$ \\
\hline
\end{tabular}

BMI (Asian Standards) were used according to the WHO/IASO/IOTF. The AsiaPacific perspective: redefining obesity and its treatment. Health communication Australia Pty Ltd; 2000. Where BMI below 18.5 is underweight; from 18.5-22.9 is normal; from 23- 24.9 is overweight; from 25-34.9 is obese Where BMI below 18.5 is underweight; from $18.5-22.9$ is normal; from $23-24.9$ is overweight; above 25 is obese

$S D$ Standard deviation, BMI Body Mass Index

questions. Percentages at or above $15 \%$ considered significant [37]. Discriminant validity was evaluated using correlations between FJS-12 final score and patients' baseline demographics. Data analysis were carried out using IBM SPSS 27.0 (Armonk, New York). A two-sided $\mathrm{p}$ value $\leq 0.05$ was considered statistically significant.

\section{Bootstrapping}

Bootstrapping was introduced to compare the differences in responsiveness estimates between the measures, and the results were expressed in terms of bias, standard error, and 95\% confidence interval (CI) [38]. Bootstrapping is a resampling technique to draw numerous samples from the original sample with replacement [39]. In this study, a bias-corrected bootstrap method (bias corrected accelerated, $\mathrm{BCa}$ ) with 200 and 1000 iterations or samples was used to compare the differences in the mentioned responsiveness estimates (In our study, bias, standard error, and 95\% confidence interval (CI) were reported) between the measures [40-42]. Two sampling sizes, 200 and 1000 were performed because 1) this was a statistics "rule of thumb" that 200 samples provide adequate statistical power for data analysis, and 2) 1000 is a presumed sample size for running bootstrapping. Bootstrapping was also carried out using IBM SPSS 27 (Armonk, New York).

\section{Results}

The baseline demographics of the 75 patients were tabulated in Table 1 . Of the 75 patients, $74.6 \%$ were obese. Mean number of days between the baseline and followup was 9.53 days. Obese patients constituted $70.67 \%$ of the 75 patients, $16 \%$ were overweight and $12 \%$ felt into normal BMI range.
Table 2 Test-retest reliability and internal consistency of FJS-12 question scores between baseline and follow-up

\begin{tabular}{lll}
\hline FJS-12 questions & ICC $(\mathbf{9 5} \% \mathbf{C I})$ & Cronbach's a \\
\hline Q1 & $0.832(0.669-0.919)$ & 0.908 \\
Q2 & $0.734(0.503-0.868)$ & 0.847 \\
Q3 & $0.517(0.184-0.743)$ & 0.681 \\
Q4 & $0.651(0.373-0.822)$ & 0.789 \\
Q5 & $0.720(0.500-0.847)$ & 0.720 \\
Q6 & $0.734(0.416-0.879)$ & 0.734 \\
Q7 & $0.745(0.440-0.884)$ & 0.745 \\
Q8 & $0.666(0.278-0.845)$ & 0.666 \\
Q9 & $0.788(0.535-0.903)$ & 0.788 \\
Q10 & $0.520(0.191-0.760)$ & 0.520 \\
Q11 & $0.839(0.647-0.927)$ & 0.839 \\
Q12 & $0.755(0.470-0.886)$ & 0.755 \\
Final score & $0.769(0.560-0.886)$ & 0.870 \\
\hline
\end{tabular}

FJS-12 Forgotten Joint Knee Score, ICC Intraclass correlation (Single measure), $\mathrm{Cl}$ Confidence interval

\section{Reliability}

FJS-12 showed moderate to excellent internal consistency in individual question with Cronbach's $\alpha$ of 0.870 in the final score (Table 2). The test-retest reliability in terms of ICC was good in the FJS-12 final score (ICC $=0.769$ $(95 \% \mathrm{CI}=0.560,0.886))$ using the definitions established by Koo et al. [43]. Question 1 was "excellent" and most of the questions indicated at least "moderate". Bland-Altman plot for the repeated measures (follow-up - baseline) showed the majority of measurement differences fell within the mean \pm 1.96 standard deviation (Fig. 1). Nearly all measurement differences fell within the $95 \%$ limits of agreement (LOA) (Fig. 1).

\section{Construct validity}

Construct validity explained by correlation analyses showed moderate correlation with OKS at baseline (FJS-12 baseline vs. OKS baseline; Pearson's coefficient $=0.598, \quad p<0.01)$ and very strong correlation at follow-up (Pearson's coefficient $=0.879, p<0.01$ ) (Table 3). Similar results were also observed in correlations between FJS-12 and NRS (moderate at baseline and very strong correlation at follow-up) (Table 4).

\section{Responsiveness}

Responsiveness in terms of SDC was 15.77. MIC was calculated by halving the standard deviation proposed by Norman et al.[44]. MIC came out to be 5.92, which was smaller than SDC (i.e., SDC was higher than MIC). Floor effect was not observable in all questions (Table 5). Ceiling effect was statistically significant in question 8 in both baseline and follow-up, unless otherwise non-specified. 


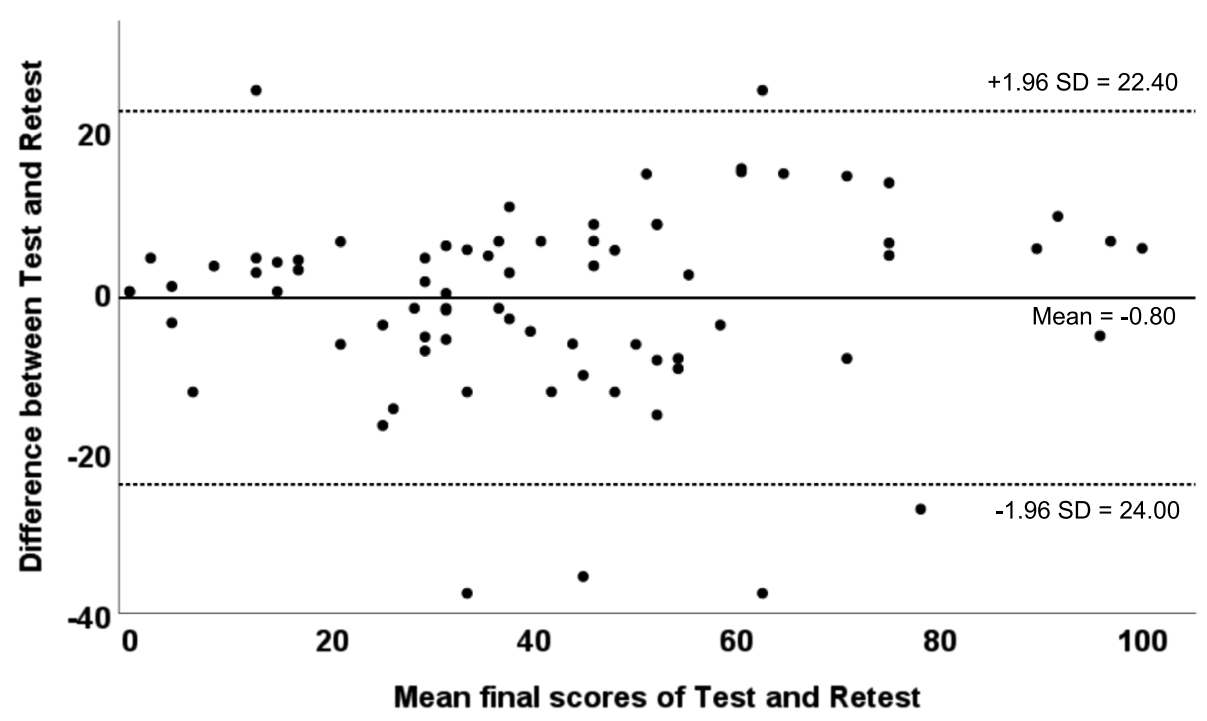

SD: Standard deviation

Fig. 1 The Bland-Altman plot for test-retest (baseline-follow-up) agreement of FJS-12.

Table 3 Correlations between FJS-12 final scores and OKS overall scores at baseline and follow-up

\begin{tabular}{llccc}
\hline FJS-12 or OKS & FJS-12 baseline & FJS-12 follow-up & OKS baseline & OKS follow-up \\
\hline FJS-12 baseline & & $0.771^{* *}$ & $0.598^{* *}$ & $0.786^{* *}$ \\
FJS-12 follow-up & $0.771^{* *}$ & & $-0.734^{* *}$ & $0.879^{* *}$ \\
OKS baseline & $0.598^{* *}$ & $-0.734^{* *}$ & $-0.848^{* *}$ \\
OKS follow-up & $0.786^{* *}$ & $0.879^{* *}$ & $-0.848^{* *}$ & \\
\hline
\end{tabular}

FJS-12 Forgotten Joint Knee Score-12 final scores, OKS Oxford Knee Score: overall scores

${ }^{* *} p<0.01$

Table 4 Correlations between FJS-12 final scores and NRS at baseline and follow-up

\begin{tabular}{llccc}
\hline FJS-12 or NRS & FJS-12 baseline & FJS-12 follow-up & NRS baseline & NRS follow-up \\
\hline FJS-12 baseline & & $0.721^{* *}$ & $0.601^{* *}$ & $0.799^{* *}$ \\
FJS-12 follow-up & $0.721^{* *}$ & & $-0.765^{* *}$ & $0.873^{* *}$ \\
NRS baseline & $0.601^{* *}$ & $-0.765^{* *}$ & $-0.837^{* *}$ \\
NRS follow-up & $0.799^{* *}$ & $0.873^{* *}$ & $-0.837^{* *}$ & \\
\hline
\end{tabular}

FJS-12 Forgotten Joint Knee Score-12 final scores, NRS Numeric Rating Scale

${ }^{* *} p<0.01$

\section{Discriminant validity}

FJS-12 baseline and follow-up were found to have no significant correlation with patients' age, sex, BMI, and side of surgery (Table 5). OKS baseline and follow-up were also put in line with the analysis and results also showed no significant correlation with the respective baseline demographics.

\section{Bootstrapping}

Bias and standard error of the mean and standard deviation of individual questions as well as total score at baseline and follow-up were both low after performing bootstrapping for 200 samples (Table 6). Similar results (low bias and standard error) were found after performing bootstrapping for 1000 samples (Table 6). In OKS, 
Table 5 Percentages of floor and ceiling effects in individual Chinese Cantonese (Hong Kong) translated FJS-12 questions collected at baseline and follow-up

\begin{tabular}{|c|c|c|c|c|}
\hline Questions & Time point & Mean \pm SD; Median & $\begin{array}{l}\text { Floor percentage (scored } \\
\text { "mostly"; \%) }\end{array}$ & $\begin{array}{l}\text { Ceiling percentage } \\
\text { (scored "never"; \%) }\end{array}$ \\
\hline \multirow[t]{2}{*}{ Q1 } & Baseline & $3.15 \pm 1.19 ; 3.00$ & 14.7 & 12.0 \\
\hline & Follow-up & $3.43 \pm 1.14 ; 4.00$ & 10.7 & 14.3 \\
\hline \multirow[t]{2}{*}{ Q2 } & Baseline & $3.13 \pm 1.26 ; 3.00$ & 14.7 & 12.0 \\
\hline & Follow-up & $3.18 \pm 1.25 ; 3.00$ & 14.3 & 14.3 \\
\hline \multirow[t]{2}{*}{ Q3 } & Baseline & $3.29 \pm 1.39 ; 4.00$ & 13.7 & 24.7 \\
\hline & Follow-up & $3.04 \pm 1.37 ; 3.00$ & 17.9 & 17.9 \\
\hline \multirow[t]{2}{*}{ Q4 } & Baseline & $2.63 \pm 1.46 ; 2.00$ & 32.0 & 13.3 \\
\hline & Follow-up & $2.93 \pm 1.44 ; 3.00$ & 25.0 & 17.9 \\
\hline \multirow[t]{2}{*}{ Q5 } & Baseline & $3.06 \pm 1.39 ; 3.00$ & 20.8 & 15.3 \\
\hline & Follow-up & $3.29 \pm 1.21 ; 3.50$ & 10.7 & 14.3 \\
\hline \multirow[t]{2}{*}{ Q6 } & Baseline & $3.92 \pm 1.13 ; 4.00$ & 6.8 & 32.9 \\
\hline & Follow-up & $3.96 \pm 1.00 ; 4.00$ & 3.6 & 35.7 \\
\hline \multirow[t]{2}{*}{ Q7 } & Baseline & $3.37 \pm 1.34 ; 4.00$ & 15.1 & 23.3 \\
\hline & Follow-up & $3.43 \pm 1.17 ; 4.00$ & 10.7 & 10.7 \\
\hline \multirow[t]{2}{*}{ Q8 } & Baseline & $3.88 \pm 1.13 ; 4.00$ & 6.7 & $32.0^{*}$ \\
\hline & Follow-up & $4.18 \pm 0.95 ; 4.00$ & 3.6 & $42.9^{*}$ \\
\hline \multirow[t]{2}{*}{ Q9 } & Baseline & $3.60 \pm 1.37 ; 4.00$ & 13.7 & 31.5 \\
\hline & Follow-up & $3.82 \pm 1.06 ; 4.00$ & 7.1 & 21.4 \\
\hline \multirow[t]{2}{*}{ Q10 } & Baseline & $3.53 \pm 1.17 ; 4.00$ & 8.2 & 20.5 \\
\hline & Follow-up & $3.54 \pm 1.11 ; 4.00$ & 10.7 & 14.3 \\
\hline \multirow[t]{2}{*}{ Q11 } & Baseline & $3.25 \pm 1.25 ; 3.00$ & 13.7 & 16.4 \\
\hline & Follow-up & $3.36 \pm 1.16 ; 4.00$ & 10.7 & 10.7 \\
\hline \multirow[t]{2}{*}{ Q12 } & Baseline & $3.33 \pm 1.32 ; 4.00$ & 14.7 & 21.3 \\
\hline & Follow-up & $3.50 \pm 1.17 ; 4.00$ & 7.1 & 17.9 \\
\hline
\end{tabular}

* Comparisons with statistical difference, $p<0.01$

Table 6 Correlation between FJS-12 final scores and participants' characteristics, and between OKS and participants' characteristics

\begin{tabular}{lllll}
\hline & Age & Sex & BMI & Side of surgery \\
\hline FJS-12 (baseline) & 0.160 & -0.153 & -0.086 & -0.018 \\
FJS-12 (follow-up) & 0.099 & -0.153 & -0.180 & -0.105 \\
OKS (baseline) & 0.097 & -0.154 & 0.113 & 0.120 \\
OKS (follow-up) & 0.119 & -0.080 & -0.325 & -0.043 \\
\hline
\end{tabular}

BMI Body Mass Index, FJS Forgotten joint score, OKS Oxford Knee Score

bias and standard error were low similar to that in FJS12 (Table 7). Table 8 showed the results of mean differences, correlation coefficients, and p values in FJS-12 and OKS after bootstrapping for 200 and 1000 samples. The calculations were based on the score differences between baseline and follow-up. In mean difference, the 95\% CI after bootstrapping for 200 and 1000 samples were similar (for example, in the comparison of mean difference in FJS-12 Question 1 between baseline and follow-up: within -0.46 and 0.00 in bootstrapping $N=200$, and within -0.42 and 0.00 in bootstrapping $N=1000$ ). Similarly, the $95 \% \mathrm{CI}$ of correlation coefficients after bootstrapping $N=200$ and $\mathrm{N}=1000$ were similar (Table 8; FJS-12 Question 1 Baseline - FJS-12 Question 1 Followup; bootstrapping for $N=200$ : $0.73-0.95$; bootstrapping for $N=1000: 0.70-0.95)$, in both FJS-12 and OKS. The $p$ values without bootstrapping and bootstrapping for $N=200$ and $N=1000$ were similar. The $p$ values showing 
Table 7 Means and standard deviations of FJS-12 individual questionnaires and total scores at baseline and follow-up, and bias, its standard error and 95\% confidence intervals after bootstrapping for $N=200$ and $N=1000$

\begin{tabular}{|c|c|c|c|c|c|c|c|c|c|}
\hline \multirow{2}{*}{$\begin{array}{l}\text { Questionnaire } \\
\text { and timepoint }\end{array}$} & \multirow{2}{*}{$\begin{array}{l}\text { Mean or } \\
\text { Standard } \\
\text { deviation }\end{array}$} & \multicolumn{4}{|c|}{ Bootstrapping $N=200$} & \multicolumn{4}{|c|}{ Bootstrapping $N=1000$} \\
\hline & & This study & Bias & $\begin{array}{l}\text { Standard } \\
\text { error }\end{array}$ & $95 \% \mathrm{Cl}$ & This study & Bias & $\begin{array}{l}\text { Standard } \\
\text { error }\end{array}$ & $95 \% \mathrm{Cl}$ \\
\hline \multirow{2}{*}{$\begin{array}{l}\text { FJS-12 Q01 } \\
\text { Baseline }\end{array}$} & Mean & 3.17 & -0.02 & 0.24 & $2.75-3.50$ & 3.17 & 0.01 & 0.22 & $2.75-3.58$ \\
\hline & $\begin{array}{l}\text { Standard } \\
\text { deviation }\end{array}$ & 1.17 & -0.00 & 0.22 & $0.88-1.43$ & 1.17 & -0.03 & 0.16 & $0.88-1.39$ \\
\hline \multirow{2}{*}{$\begin{array}{l}\text { FJS-12 Q01 } \\
\text { Follow-up }\end{array}$} & Mean & 3.38 & -0.03 & 0.22 & $3.00-3.67$ & 3.38 & 0.01 & 0.23 & $2.92-3.79$ \\
\hline & $\begin{array}{l}\text { Standard } \\
\text { deviation }\end{array}$ & 1.17 & -0.02 & 0.15 & $0.88-1.41$ & 1.17 & -0.04 & 0.17 & $0.84-1.38$ \\
\hline \multirow{2}{*}{$\begin{array}{l}\text { FJS-12 Q02 } \\
\text { Baseline }\end{array}$} & Mean & 3.33 & 0.01 & 0.25 & $2.88-3.87$ & 3.33 & 0.01 & 0.24 & $2.88-3.79$ \\
\hline & $\begin{array}{l}\text { Standard } \\
\text { deviation }\end{array}$ & 1.20 & -0.05 & 0.15 & $0.93-1.37$ & 1.20 & -0.04 & 0.14 & $0.98-1.34$ \\
\hline \multirow{2}{*}{$\begin{array}{l}\text { FJS-12 Q02 } \\
\text { Follow-up }\end{array}$} & Mean & 3.25 & 0.00 & 0.26 & $2.79-3.67$ & 3.25 & 0.01 & 0.25 & $2.75-3.75$ \\
\hline & $\begin{array}{l}\text { Standard } \\
\text { deviation }\end{array}$ & 1.26 & -0.05 & 0.15 & $1.01-1.42$ & 1.26 & -0.04 & 0.14 & $1.01-1.41$ \\
\hline \multirow{2}{*}{$\begin{array}{l}\text { FJS-12 Q03 } \\
\text { Baseline }\end{array}$} & Mean & 3.29 & -0.02 & 0.28 & $2.75-3.72$ & 3.29 & 0.01 & 0.27 & $2.75-3.79$ \\
\hline & $\begin{array}{l}\text { Standard } \\
\text { deviation }\end{array}$ & 1.37 & -0.04 & 0.13 & $1.20-1.47$ & 1.37 & -0.04 & 0.13 & $1.14-1.52$ \\
\hline \multirow{2}{*}{$\begin{array}{l}\text { FJS-12 Q03 } \\
\text { Follow-up }\end{array}$} & Mean & 3.13 & 0.00 & 0.28 & $2.67-3.62$ & 3.13 & 0.01 & 0.27 & $2.63-3.67$ \\
\hline & $\begin{array}{l}\text { Standard } \\
\text { deviation }\end{array}$ & 1.39 & -0.04 & 0.14 & $1.19-1.51$ & 1.39 & -0.04 & 0.13 & $1.16-1.54$ \\
\hline \multirow{2}{*}{$\begin{array}{l}\text { FJS-12 Q04 } \\
\text { Baseline }\end{array}$} & Mean & 2.63 & 0.00 & 0.29 & $2.17-3.17$ & 2.63 & 0.01 & 0.28 & $2.04-3.13$ \\
\hline & $\begin{array}{l}\text { Standard } \\
\text { deviation }\end{array}$ & 1.44 & -0.02 & 0.10 & $1.23-1.59$ & 1.44 & -0.03 & 0.12 & $1.25-1.58$ \\
\hline \multirow{2}{*}{$\begin{array}{l}\text { FJS-12 Q04 } \\
\text { Follow-up }\end{array}$} & Mean & 3.00 & 0.03 & 0.29 & $2.37-3.59$ & 3.00 & 0.02 & 0.30 & $2.38-3.63$ \\
\hline & $\begin{array}{l}\text { Standard } \\
\text { deviation }\end{array}$ & 1.47 & -0.05 & 0.13 & $1.30-1.55$ & 1.47 & -0.04 & 0.14 & $1.27-1.60$ \\
\hline \multirow{2}{*}{$\begin{array}{l}\text { FJS-12 Q05 } \\
\text { Baseline }\end{array}$} & Mean & 2.96 & 0.01 & 0.27 & $2.46-3.46$ & 2.96 & 0.02 & 0.27 & $2.42-3.50$ \\
\hline & $\begin{array}{l}\text { Standard } \\
\text { deviation }\end{array}$ & 1.40 & -0.03 & 0.13 & $1.14-1.56$ & 1.40 & -0.03 & 0.13 & $1.18-1.53$ \\
\hline \multirow{2}{*}{$\begin{array}{l}\text { FJS-12 Q05 } \\
\text { Follow-up }\end{array}$} & Mean & 3.21 & -0.01 & 0.24 & $2.79-3.67$ & 3.21 & 0.01 & 0.24 & $2.79-3.63$ \\
\hline & $\begin{array}{l}\text { Standard } \\
\text { deviation }\end{array}$ & 1.22 & -0.04 & 0.14 & $0.97-1.38$ & 1.22 & -0.03 & 0.14 & $0.98-1.38$ \\
\hline \multirow{2}{*}{$\begin{array}{l}\text { FJS-12 Q06 } \\
\text { Baseline }\end{array}$} & Mean & 3.88 & 0.00 & 0.23 & $3.50-4.25$ & 3.88 & 0.01 & 0.23 & $3.42-4.33$ \\
\hline & $\begin{array}{l}\text { Standard } \\
\text { deviation }\end{array}$ & 1.19 & -0.04 & 0.20 & $0.78-1.47$ & 1.19 & -0.05 & 0.20 & $0.81-1.44$ \\
\hline \multirow{2}{*}{$\begin{array}{l}\text { FJS-12 Q06 } \\
\text { Follow-up }\end{array}$} & Mean & 3.92 & -0.02 & 0.18 & $3.67-4.17$ & 3.92 & 0.00 & 0.20 & $3.54-4.29$ \\
\hline & $\begin{array}{l}\text { Standard } \\
\text { deviation }\end{array}$ & 1.02 & -0.03 & 0.15 & $0.76-1.24$ & 1.02 & -0.04 & 0.17 & $0.75-1.23$ \\
\hline \multirow{2}{*}{$\begin{array}{l}\text { FJS-12 Q07 } \\
\text { Baseline }\end{array}$} & Mean & 3.25 & 0.01 & 0.24 & $2.88-3.75$ & 3.25 & 0.01 & 0.24 & $2.79-3.67$ \\
\hline & $\begin{array}{l}\text { Standard } \\
\text { deviation }\end{array}$ & 1.19 & -0.03 & 0.14 & $0.96-1.35$ & 1.19 & -0.04 & 0.15 & $0.92-1.36$ \\
\hline \multirow{2}{*}{$\begin{array}{l}\text { FJS-12 Q07 } \\
\text { Follow-up }\end{array}$} & Mean & 3.54 & 0.02 & 0.24 & $3.06-4.03$ & 3.54 & 0.01 & 0.23 & $3.04-4.00$ \\
\hline & $\begin{array}{l}\text { Standard } \\
\text { deviation }\end{array}$ & 1.18 & -0.07 & 0.21 & $0.75-1.40$ & 1.18 & -0.05 & 0.20 & $0.83-1.38$ \\
\hline \multirow{2}{*}{$\begin{array}{l}\text { FJS-12 Q08 } \\
\text { Baseline }\end{array}$} & Mean & 3.75 & 0.00 & 0.23 & $3.29-4.13$ & 3.75 & 0.01 & 0.23 & $3.29-4.21$ \\
\hline & $\begin{array}{l}\text { Standard } \\
\text { deviation }\end{array}$ & 1.19 & -0.04 & 0.17 & $0.86-1.44$ & 1.19 & -0.04 & 0.18 & $0.88-1.38$ \\
\hline \multirow{2}{*}{$\begin{array}{l}\text { FJS-12 Q08 } \\
\text { Follow-up }\end{array}$} & Mean & 4.13 & 0.00 & 0.19 & $3.79-4.50$ & 4.13 & 0.00 & 0.20 & $3.75-4.50$ \\
\hline & $\begin{array}{l}\text { Standard } \\
\text { deviation }\end{array}$ & 0.99 & -0.05 & 0.19 & $0.66-1.20$ & 0.99 & -0.04 & 0.20 & $0.66-1.23$ \\
\hline
\end{tabular}


Table 7 (continued)

\begin{tabular}{|c|c|c|c|c|c|c|c|c|c|}
\hline \multirow{2}{*}{$\begin{array}{l}\text { Questionnaire } \\
\text { and timepoint }\end{array}$} & \multirow{2}{*}{$\begin{array}{l}\text { Mean or } \\
\text { Standard } \\
\text { deviation }\end{array}$} & \multicolumn{4}{|c|}{ Bootstrapping $N=200$} & \multicolumn{4}{|c|}{ Bootstrapping $N=1000$} \\
\hline & & This study & Bias & $\begin{array}{l}\text { Standard } \\
\text { error }\end{array}$ & $95 \% \mathrm{Cl}$ & This study & Bias & $\begin{array}{l}\text { Standard } \\
\text { error }\end{array}$ & $95 \% \mathrm{Cl}$ \\
\hline \multirow{2}{*}{$\begin{array}{l}\text { FJS-12 Q09 } \\
\text { Baseline }\end{array}$} & Mean & 3.92 & 0.00 & 0.20 & $3.54-4.25$ & 3.92 & 0.01 & 0.22 & $3.50-4.33$ \\
\hline & $\begin{array}{l}\text { Standard } \\
\text { deviation }\end{array}$ & 1.10 & -0.02 & 0.17 & $0.76-1.35$ & 1.10 & -0.04 & 0.18 & $0.76-1.34$ \\
\hline \multirow{2}{*}{$\begin{array}{l}\text { FJS-12 Q09 } \\
\text { Follow-up }\end{array}$} & Mean & 4.00 & 0.00 & 0.16 & $3.74-4.25$ & 4.00 & 0.00 & 0.18 & $3.67-4.29$ \\
\hline & $\begin{array}{l}\text { Standard } \\
\text { deviation }\end{array}$ & 0.89 & -0.04 & 0.20 & $0.55-1.12$ & 0.89 & -0.04 & 0.22 & $0.54-1.14$ \\
\hline \multirow{2}{*}{$\begin{array}{l}\text { FJS-12 Q10 } \\
\text { Baseline }\end{array}$} & Mean & 3.63 & -0.01 & 0.20 & $3.29-3.96$ & 3.63 & 0.01 & 0.22 & $3.17-4.08$ \\
\hline & $\begin{array}{l}\text { Standard } \\
\text { deviation }\end{array}$ & 1.14 & -0.04 & 0.17 & $0.79-1.37$ & 1.14 & -0.04 & 0.18 & $0.83-1.34$ \\
\hline \multirow{2}{*}{$\begin{array}{l}\text { FJS-12 Q10 } \\
\text { Follow-up }\end{array}$} & Mean & 3.58 & 0.01 & 0.19 & $3.17-3.92$ & 3.58 & 0.00 & 0.21 & $3.21-3.96$ \\
\hline & $\begin{array}{l}\text { Standard } \\
\text { deviation }\end{array}$ & 1.06 & -0.06 & 0.18 & $0.65-1.26$ & 1.06 & -0.04 & 0.19 & $0.70-1.29$ \\
\hline \multirow{2}{*}{$\begin{array}{l}\text { FJS-12 Q11 } \\
\text { Baseline }\end{array}$} & Mean & 3.33 & -0.01 & 0.20 & $3.00-3.67$ & 3.33 & 0.01 & 0.22 & $2.92-3.75$ \\
\hline & $\begin{array}{l}\text { Standard } \\
\text { deviation }\end{array}$ & 1.09 & -0.03 & 0.15 & $0.78-1.31$ & 1.09 & -0.04 & 0.15 & $0.83-1.25$ \\
\hline \multirow{2}{*}{$\begin{array}{l}\text { FJS-12 Q11 } \\
\text { Follow-up }\end{array}$} & Mean & 3.50 & 0.01 & 0.21 & $3.13-3.88$ & 3.50 & 0.01 & 0.22 & $3.08-3.92$ \\
\hline & $\begin{array}{l}\text { Standard } \\
\text { deviation }\end{array}$ & 1.10 & -0.05 & 0.17 & $0.76-1.29$ & 1.10 & -0.05 & 0.17 & $0.85-1.25$ \\
\hline \multirow{2}{*}{$\begin{array}{l}\text { FJS-12 Q12 } \\
\text { Baseline }\end{array}$} & Mean & 3.29 & 0.00 & 0.20 & $2.98-3.67$ & 3.29 & 0.01 & 0.22 & $2.88-3.71$ \\
\hline & $\begin{array}{l}\text { Standard } \\
\text { deviation }\end{array}$ & 1.08 & -0.03 & 0.15 & $0.78-1.32$ & 1.08 & -0.04 & 0.15 & $0.83-1.25$ \\
\hline \multirow{2}{*}{$\begin{array}{l}\text { FJS-12 Q12 } \\
\text { Follow-up }\end{array}$} & Mean & 3.50 & 0.02 & 0.24 & $3.00-4.02$ & 3.50 & 0.02 & 0.25 & $2.97-4.04$ \\
\hline & $\begin{array}{l}\text { Standard } \\
\text { deviation }\end{array}$ & 1.25 & -0.05 & 0.15 & $1.00-1.40$ & 1.25 & -0.04 & 0.15 & $1.03-1.38$ \\
\hline \multirow{2}{*}{$\begin{array}{l}\text { FJS-12Total } \\
\text { Baseline }\end{array}$} & Mean & 40.80 & 0.06 & 4.99 & $29.55-50.70$ & 40.80 & -0.31 & 4.91 & $32.47-48.70$ \\
\hline & $\begin{array}{l}\text { Standard } \\
\text { deviation }\end{array}$ & 25.28 & -0.56 & 3.32 & $18.26-30.57$ & 25.28 & -0.76 & 3.36 & $19.54-29.29$ \\
\hline \multirow{2}{*}{$\begin{array}{l}\text { FJS-12 Total } \\
\text { Follow-up }\end{array}$} & Mean & 37.24 & -0.06 & 4.46 & $29.13-46.58$ & 37.24 & -0.23 & 4.68 & $28.65-45.58$ \\
\hline & $\begin{array}{l}\text { Standard } \\
\text { deviation }\end{array}$ & 23.63 & -1.00 & 3.51 & $17.08-27.17$ & 23.63 & -0.75 & 3.70 & $17.55-28.08$ \\
\hline
\end{tabular}

non-statistical significance $(p>0.05)$ without bootstrapping remained statistical insignificance after bootstrapping of both sampling sizes. In the comparison group "OKS Question 12 Baseline - OKS Question 12 Followup", the score difference was found to have statistical significance $(p=0.05)$. Statistical difference remained after the two bootstrapping methods ( $p=0.02$ after bootstrapping for $N=200$; and $p=0.05$ after bootstrapping for $N=1000)$.

Cross-comparisons between FJS-12 and OKS individual scores at baseline and follow-up followed. In the comparisons between FJS-12 and OKS in the 13 individual questions (12 questions and total) at baseline, mean differences and correlation coefficients were similar (Table 9). These results were reflected by the $\mathrm{p}$ values without bootstrapping, bootstrapping for $N=200$, and bootstrapping for $N=1000$ (Table 10). Comparing between $95 \%$ CI of mean difference and $95 \%$ CI of correlation coefficient in FJS-12 and OKS after bootstrapping for $N=200$ and for $N=100$ showed similar results (Table 11). For example, in the comparison "FJS-12 Q01 Follow-up - OKS Q01 Follow-up", the 95\% CI of mean differences were 0.43 to 2.14 (bootstrapping for $N=200$ ) and 0.43 to 2.14 (bootstrapping for $N=1000$ ) (Table 11, first row). The $p$ values were 0.01 (without bootstrapping), 0.02 (bootstrapping for $N=200$ ), and 0.01 (bootstrapping for $N=1000$ ). Comparisons showing statistical significance (i.e. $p<0.05$ ) without applying bootstrapping remained statistically significant after bootstrapping for $N=200$ and for $N=1000$. This was reflected in comparisons "FJS-12 Q06 Follow-up - OKS Q06 Follow-up", "FJS-12 Q07 
Table 8 Means and standard deviations of OKS individual questions and total scores at baseline and follow-up, and bias, its standard error and 95\% confidence intervals after bootstrapping for $N=200$ and $N=1000$

\begin{tabular}{|c|c|c|c|c|c|c|c|c|c|}
\hline \multirow{2}{*}{$\begin{array}{l}\text { Question and } \\
\text { timepoint }\end{array}$} & \multirow{2}{*}{$\begin{array}{l}\text { Mean or } \\
\text { Standard } \\
\text { deviation }\end{array}$} & \multicolumn{4}{|c|}{ Bootstrapping $N=200$} & \multicolumn{4}{|c|}{ Bootstrapping $N=1000$} \\
\hline & & This study & Bias & $\begin{array}{l}\text { Standard } \\
\text { error }\end{array}$ & $95 \% \mathrm{Cl}$ & This study & Bias & $\begin{array}{l}\text { Standard } \\
\text { error }\end{array}$ & $95 \% \mathrm{Cl}$ \\
\hline \multirow{2}{*}{$\begin{array}{l}\text { OKS Q01 } \\
\text { Baseline }\end{array}$} & Mean & 2.22 & 0.03 & 0.23 & $1.81-2.67$ & 2.22 & 0.00 & 0.23 & $1.81-2.63$ \\
\hline & $\begin{array}{l}\text { Standard } \\
\text { deviation }\end{array}$ & 1.22 & -0.02 & 0.12 & $0.99-1.37$ & 1.22 & -0.02 & 0.14 & $0.94-1.42$ \\
\hline \multirow{2}{*}{$\begin{array}{l}\text { OKS Q01 } \\
\text { Follow-up }\end{array}$} & Mean & 2.19 & 0.03 & 0.23 & $1.81-2.63$ & 2.19 & 0.00 & 0.23 & $1.81-2.56$ \\
\hline & $\begin{array}{l}\text { Standard } \\
\text { deviation }\end{array}$ & 1.21 & -0.02 & 0.11 & $1.04-1.35$ & 1.21 & -0.02 & 0.13 & $1.00-1.36$ \\
\hline \multirow{2}{*}{$\begin{array}{l}\text { OKS Q02 } \\
\text { Baseline }\end{array}$} & Mean & 3.07 & 0.01 & 0.17 & $2.78-3.38$ & 3.07 & -0.01 & 0.16 & $2.81-3.33$ \\
\hline & $\begin{array}{l}\text { Standard } \\
\text { deviation }\end{array}$ & 0.83 & -0.03 & 0.11 & $0.68-0.94$ & 0.83 & -0.02 & 0.11 & $0.65-0.96$ \\
\hline \multirow{2}{*}{$\begin{array}{l}\text { OKS Q02 } \\
\text { Follow-up }\end{array}$} & Mean & 3.00 & 0.00 & 0.18 & $2.70-3.30$ & 3.00 & 0.00 & 0.18 & $2.67-3.26$ \\
\hline & $\begin{array}{l}\text { Standard } \\
\text { deviation }\end{array}$ & 0.92 & -0.02 & 0.17 & $0.62-1.18$ & 0.92 & -0.04 & 0.18 & $0.63-1.14$ \\
\hline \multirow{2}{*}{$\begin{array}{l}\text { OKS Q03 } \\
\text { Baseline }\end{array}$} & Mean & 2.78 & 0.01 & 0.18 & $2.46-3.10$ & 2.78 & 0.00 & 0.17 & $2.52-3.07$ \\
\hline & $\begin{array}{l}\text { Standard } \\
\text { deviation }\end{array}$ & 0.89 & -0.02 & 0.09 & $0.75-1.00$ & 0.89 & -0.02 & 0.10 & $0.71-1.01$ \\
\hline \multirow{2}{*}{$\begin{array}{l}\text { OKS Q03 } \\
\text { Follow-up }\end{array}$} & Mean & 2.74 & 0.01 & 0.20 & $2.40-3.12$ & 2.74 & -0.01 & 0.18 & $2.44-3.04$ \\
\hline & $\begin{array}{l}\text { Standard } \\
\text { deviation }\end{array}$ & 0.94 & -0.02 & 0.10 & $0.79-1.07$ & 0.94 & -0.02 & 0.09 & $0.80-1.05$ \\
\hline \multirow{2}{*}{$\begin{array}{l}\text { OKS Q04 } \\
\text { Baseline }\end{array}$} & Mean & 3.33 & 0.02 & 0.18 & $2.96-3.72$ & 3.33 & 0.00 & 0.18 & $3.04-3.59$ \\
\hline & $\begin{array}{l}\text { Standard } \\
\text { deviation }\end{array}$ & 0.92 & -0.05 & 0.12 & $0.70-1.01$ & 0.92 & -0.02 & 0.12 & $0.69-1.10$ \\
\hline \multirow{2}{*}{$\begin{array}{l}\text { OKS Q04 } \\
\text { Follow-up }\end{array}$} & Mean & 3.26 & 0.02 & 0.14 & $3.00-3.59$ & 3.26 & 0.00 & 0.16 & $3.00-3.52$ \\
\hline & $\begin{array}{l}\text { Standard } \\
\text { deviation }\end{array}$ & 0.81 & -0.02 & 0.08 & $0.69-0.89$ & 0.81 & -0.02 & 0.07 & $0.68-0.90$ \\
\hline \multirow{2}{*}{$\begin{array}{l}\text { OKS Q05 } \\
\text { Baseline }\end{array}$} & Mean & 2.89 & 0.02 & 0.17 & $2.59-3.27$ & 2.89 & 0.00 & 0.16 & $2.63-3.15$ \\
\hline & $\begin{array}{l}\text { Standard } \\
\text { deviation }\end{array}$ & 0.85 & -0.03 & 0.09 & $0.71-0.93$ & 0.85 & -0.02 & 0.09 & $0.71-0.94$ \\
\hline \multirow{2}{*}{$\begin{array}{l}\text { OKS Q05 } \\
\text { Follow-up }\end{array}$} & Mean & 2.78 & 0.03 & 0.16 & $2.44-3.19$ & 2.78 & 0.00 & 0.17 & $2.48-3.04$ \\
\hline & $\begin{array}{l}\text { Standard } \\
\text { deviation }\end{array}$ & 0.89 & -0.03 & 0.11 & $0.71-1.01$ & 0.89 & -0.02 & 0.12 & $0.65-1.05$ \\
\hline \multirow{2}{*}{$\begin{array}{l}\text { OKS Q06 } \\
\text { Baseline }\end{array}$} & Mean & 2.78 & 0.02 & 0.24 & $2.33-3.27$ & 2.78 & 0.00 & 0.23 & $2.44-3.15$ \\
\hline & $\begin{array}{l}\text { Standard } \\
\text { deviation }\end{array}$ & 1.22 & -0.03 & 0.14 & $0.96-1.41$ & 1.22 & -0.03 & 0.14 & $0.92-1.42$ \\
\hline \multirow{2}{*}{$\begin{array}{l}\text { OKS Q06 } \\
\text { Follow-up }\end{array}$} & Mean & 2.74 & 0.01 & 0.22 & $2.36-3.15$ & 2.74 & 0.00 & 0.22 & $2.37-3.11$ \\
\hline & $\begin{array}{l}\text { Standard } \\
\text { deviation }\end{array}$ & 1.23 & -0.02 & 0.16 & $0.89-1.50$ & 1.23 & -0.02 & 0.16 & $0.88-1.48$ \\
\hline \multirow{2}{*}{$\begin{array}{l}\text { OKS Q07 } \\
\text { Baseline }\end{array}$} & Mean & 1.22 & 0.01 & 0.28 & $0.63-1.82$ & 1.22 & 0.00 & 0.27 & $0.70-1.76$ \\
\hline & $\begin{array}{l}\text { Standard } \\
\text { deviation }\end{array}$ & 1.42 & -0.02 & 0.14 & $1.16-1.64$ & 1.42 & -0.03 & 0.15 & $1.16-1.60$ \\
\hline \multirow{2}{*}{$\begin{array}{l}\text { OKS Q07 } \\
\text { Follow-up }\end{array}$} & Mean & 1.07 & 0.01 & 0.27 & $0.58-1.64$ & 1.07 & 0.00 & 0.28 & $0.63-1.59$ \\
\hline & $\begin{array}{l}\text { Standard } \\
\text { deviation }\end{array}$ & 1.44 & -0.02 & 0.15 & $1.11-1.66$ & 1.44 & -0.04 & 0.16 & $1.14-1.62$ \\
\hline \multirow{2}{*}{$\begin{array}{l}\text { OKS Q08 } \\
\text { Baseline }\end{array}$} & Mean & 2.85 & 0.01 & 0.21 & $2.48-3.24$ & 2.85 & 0.00 & 0.21 & $2.52-3.19$ \\
\hline & $\begin{array}{l}\text { Standard } \\
\text { deviation }\end{array}$ & 1.10 & -0.03 & 0.13 & $0.86-1.23$ & 1.10 & -0.02 & 0.12 & $0.88-1.25$ \\
\hline \multirow{2}{*}{$\begin{array}{l}\text { OKS Q08 } \\
\text { Follow-up }\end{array}$} & Mean & 2.74 & 0.03 & 0.22 & $2.33-3.23$ & 2.74 & -0.01 & 0.23 & $2.30-3.11$ \\
\hline & $\begin{array}{l}\text { Standard } \\
\text { deviation }\end{array}$ & 1.20 & -0.04 & 0.14 & $0.95-1.36$ & 1.20 & -0.02 & 0.13 & $0.94-1.39$ \\
\hline
\end{tabular}


Table 8 (continued)

\begin{tabular}{|c|c|c|c|c|c|c|c|c|c|}
\hline \multirow{2}{*}{$\begin{array}{l}\text { Question and } \\
\text { timepoint }\end{array}$} & \multirow{2}{*}{$\begin{array}{l}\text { Mean or } \\
\text { Standard } \\
\text { deviation }\end{array}$} & \multicolumn{4}{|c|}{ Bootstrapping $N=200$} & \multicolumn{4}{|c|}{ Bootstrapping $N=1000$} \\
\hline & & This study & Bias & $\begin{array}{l}\text { Standard } \\
\text { error }\end{array}$ & $95 \% \mathrm{Cl}$ & This study & Bias & $\begin{array}{l}\text { Standard } \\
\text { error }\end{array}$ & $95 \% \mathrm{Cl}$ \\
\hline \multirow{2}{*}{$\begin{array}{l}\text { OKS Q09 } \\
\text { Baseline }\end{array}$} & Mean & 2.52 & 0.02 & 0.19 & $2.19-2.89$ & 2.52 & 0.00 & 0.18 & $2.22-2.81$ \\
\hline & $\begin{array}{l}\text { Standard } \\
\text { deviation }\end{array}$ & 0.98 & -0.02 & 0.09 & $0.81-1.08$ & 0.98 & -0.02 & 0.11 & $0.82-1.09$ \\
\hline \multirow{2}{*}{$\begin{array}{l}\text { OKS Q09 } \\
\text { Follow-up }\end{array}$} & Mean & 2.52 & 0.01 & 0.18 & $2.19-2.86$ & 2.52 & -0.01 & 0.18 & $2.22-2.78$ \\
\hline & $\begin{array}{l}\text { Standard } \\
\text { deviation }\end{array}$ & 0.94 & -0.03 & 0.14 & $0.64-1.12$ & 0.94 & -0.03 & 0.14 & $0.68-1.14$ \\
\hline \multirow{2}{*}{$\begin{array}{l}\text { OKS Q10 } \\
\text { Baseline }\end{array}$} & Mean & 2.93 & 0.00 & 0.18 & $2.67-3.26$ & 2.93 & 0.00 & 0.18 & $2.63-3.22$ \\
\hline & $\begin{array}{l}\text { Standard } \\
\text { deviation }\end{array}$ & 0.96 & -0.02 & 0.12 & $0.70-1.13$ & 0.96 & -0.02 & 0.12 & $0.71-1.13$ \\
\hline \multirow{2}{*}{$\begin{array}{l}\text { OKS Q10 } \\
\text { Follow-up }\end{array}$} & Mean & 3.00 & 0.01 & 0.17 & $2.70-3.30$ & 3.00 & 0.01 & 0.17 & $2.70-3.30$ \\
\hline & $\begin{array}{l}\text { Standard } \\
\text { deviation }\end{array}$ & 0.88 & -0.03 & 0.13 & $0.64-1.03$ & 0.88 & -0.02 & 0.12 & $0.65-1.04$ \\
\hline \multirow{2}{*}{$\begin{array}{l}\text { OKS Q11 } \\
\text { Baseline }\end{array}$} & Mean & 2.93 & 0.01 & 0.27 & $2.37-3.43$ & 2.93 & -0.01 & 0.23 & $2.52-3.30$ \\
\hline & $\begin{array}{l}\text { Standard } \\
\text { deviation }\end{array}$ & 1.21 & -0.04 & 0.21 & $0.86-1.44$ & 1.21 & -0.03 & 0.18 & $0.86-1.45$ \\
\hline \multirow{2}{*}{$\begin{array}{l}\text { OKS Q11 } \\
\text { Follow-up }\end{array}$} & Mean & 2.85 & 0.01 & 0.22 & $2.48-3.27$ & 2.85 & 0.00 & 0.20 & $2.52-3.19$ \\
\hline & $\begin{array}{l}\text { Standard } \\
\text { deviation }\end{array}$ & 1.06 & -0.03 & 0.11 & $0.83-1.19$ & 1.06 & -0.02 & 0.10 & $0.90-1.17$ \\
\hline \multirow{2}{*}{$\begin{array}{l}\text { OKS Q12 } \\
\text { Baseline }\end{array}$} & Mean & 2.85 & 0.01 & 0.19 & $2.44-3.22$ & 2.85 & 0.00 & 0.19 & $2.53-3.15$ \\
\hline & $\begin{array}{l}\text { Standard } \\
\text { deviation }\end{array}$ & 0.99 & -0.03 & 0.17 & $0.70-1.25$ & 0.99 & -0.03 & 0.16 & $0.71-1.22$ \\
\hline \multirow{2}{*}{$\begin{array}{l}\text { OKS Q12 } \\
\text { Follow-up }\end{array}$} & Mean & 2.44 & 0.02 & 0.25 & $1.96-3.02$ & 2.44 & 0.00 & 0.23 & $2.04-2.85$ \\
\hline & $\begin{array}{l}\text { Standard } \\
\text { deviation }\end{array}$ & 1.25 & -0.03 & 0.14 & $1.05-1.40$ & 1.25 & -0.04 & 0.15 & $1.00-1.42$ \\
\hline \multirow{2}{*}{$\begin{array}{l}\text { OKS Total } \\
\text { Baseline }\end{array}$} & Mean & 27.11 & -0.13 & 1.93 & $23.59-30.23$ & 27.11 & 0.02 & 1.82 & $23.33-30.91$ \\
\hline & $\begin{array}{l}\text { Standard } \\
\text { deviation }\end{array}$ & 9.64 & -0.17 & 0.94 & $7.98-10.78$ & 9.64 & -0.21 & 0.95 & $7.86-10.90$ \\
\hline \multirow{2}{*}{$\begin{array}{l}\text { OKS Total } \\
\text { Follow-up }\end{array}$} & Mean & 31.33 & 0.20 & 1.95 & $27.52-35.73$ & 31.33 & -0.03 & 1.90 & $27.87-34.59$ \\
\hline & $\begin{array}{l}\text { Standard } \\
\text { deviation }\end{array}$ & 9.96 & -0.17 & 0.89 & $8.17-11.31$ & 9.96 & -0.21 & 1.01 & $8.05-11.27$ \\
\hline
\end{tabular}

Follow-up - OKS Q07 Follow-up”, "FJS-12 Q08 Follow-up - OKS Q08 Follow-up", "FJS-12 Q09 Follow-up - OKS Q09 Follow-up", "FJS-12 Q12 Follow-up - OKS Q12 Follow-up",

\section{Discussion}

This study validated the Traditional Chinese-Hong Kong version of FJS-12. The 75 patients underwent TKA for at least 1 year completed the translated FJS-12 twice, about 2 weeks apart. All patients also completed OKS at the two time points serving as the gold standard. Results showed moderate to excellent reliability and validity in FJS-12, in both individual questions and final score. Relationship between the differences in mean and mean values between baseline and follow-up showed good agreement. Responsiveness was proven fine with the absence of ceiling or floor effect. Discriminant validity showed no significant correlation between final score and baseline demographical variables.

Obesity is a well-known risk factor for OA, and endstage OA patients demand for TKA. World Health Organization (WHO) released a brochure on "Global Strategy on Diet, Physical Activity and Health" in year 2004 [45] followed by a global action plan on physical activity 2018-2030 in year 2018 [46]. A recent report projected the obesity trend in 2030 that the number of people who are overweigh might reach a total of 2.16 billion and another 1.12 billion obese population, or $38 \%$ and $20 \%$ of the world's adult population respectively [47]. Mean BMI of patients in our previous studies always fell within "overweight" or "obese" categories [48-50]. Consequently, a PRO questionnaire for patients underwent TKA is 
Table 9 Summary table of the $95 \%$ Cl of mean difference, correlation coefficient, and p value between FJS-12 baseline and FJS-12 follow-up in individual questions using paired T-tests after applying bootstrapping with $N=200$ and $N=1000$

\begin{tabular}{|c|c|c|c|c|c|c|c|c|c|}
\hline \multirow{2}{*}{$\begin{array}{l}\text { Question } \\
\text { and } \\
\text { timepoint }\end{array}$} & \multirow[b]{2}{*}{$\begin{array}{l}\text { Mean } \\
\text { difference }\end{array}$} & \multirow{2}{*}{$\begin{array}{l}\text { Bootstrapping } \\
N=200 \\
95 \% \mathrm{Cl} \text { of mean } \\
\text { difference after } \\
\text { bootstrap }\end{array}$} & \multirow{2}{*}{$\begin{array}{l}\text { Bootstrapping } \\
N=1000 \\
95 \% \mathrm{Cl} \text { of mean } \\
\text { difference after } \\
\text { bootstrap }\end{array}$} & \multirow[b]{2}{*}{$\begin{array}{l}\text { Correlation } \\
\text { coefficient }\end{array}$} & \multirow{2}{*}{$\begin{array}{l}\text { Bootstrapping } \\
N=200 \\
95 \% \mathrm{Cl} \text { of } \\
\text { correlation } \\
\text { coefficient after } \\
\text { bootstrap }\end{array}$} & \multirow{2}{*}{$\begin{array}{l}\text { Bootstrapping } \\
N=1000 \\
95 \% \mathrm{Cl} \text { of } \\
\text { correlation } \\
\text { coefficient after } \\
\text { bootstrap }\end{array}$} & \multirow[b]{2}{*}{$P$ value } & \multirow{2}{*}{$\begin{array}{l}\text { Bootstrapping } \\
N=200 \\
P \text { value after } \\
\text { bootstrap }\end{array}$} & \multirow{2}{*}{$\begin{array}{l}\text { Bootstrapping } \\
N=1000 \\
P \text { value after } \\
\text { bootstrap }\end{array}$} \\
\hline & & & & & & & & & \\
\hline $\begin{array}{l}\text { FJS-12 Q01 } \\
\text { Baseline - } \\
\text { FJS-12 Q01 } \\
\text { Follow-up }\end{array}$ & -0.21 & $-0.46-0.00$ & $-0.42-0.00$ & 0.87 & $0.73-0.95$ & $0.70-0.95$ & 0.10 & 0.10 & 0.09 \\
\hline $\begin{array}{l}\text { FJS-12 Q02 } \\
\text { Baseline - } \\
\text { FJS-12 Q02 } \\
\text { Follow-up }\end{array}$ & 0.08 & $-0.19-0.36$ & $-0.13-0.29$ & 0.86 & $0.73-0.93$ & $0.70-0.94$ & 0.54 & 0.51 & 0.52 \\
\hline $\begin{array}{l}\text { FJS-12 Q03 } \\
\text { Baseline - } \\
\text { FJS-12 Q03 } \\
\text { Follow-up }\end{array}$ & 0.17 & $-0.34-0.68$ & $-0.25-0.58$ & 0.62 & $0.26-0.87$ & $0.28-0.83$ & 0.50 & 0.48 & 0.49 \\
\hline $\begin{array}{l}\text { FJS-12 Q04 } \\
\text { Baseline - } \\
\text { FJS-12 Q04 } \\
\text { Follow-up }\end{array}$ & -0.38 & $-0.90-0.15$ & $-0.83-0.00$ & 0.64 & $0.34-0.86$ & $0.25-0.86$ & 0.15 & 0.17 & 0.16 \\
\hline $\begin{array}{l}\text { FJS-12 Q05 } \\
\text { Baseline - } \\
\text { FJS-12 Q05 } \\
\text { Follow-up }\end{array}$ & -0.25 & $-0.88-0.38$ & $-0.83-0.29$ & 0.36 & $-0.11-0.72$ & $-0.08-0.74$ & 0.42 & 0.41 & 0.42 \\
\hline $\begin{array}{l}\text { FJS-12 Q06 } \\
\text { Baseline - } \\
\text { FJS-12 Q06 } \\
\text { Follow-up }\end{array}$ & -0.04 & $-0.46-0.38$ & $-0.46-0.33$ & 0.60 & $0.00-0.95$ & $-0.12-0.96$ & 0.84 & 0.88 & 0.85 \\
\hline $\begin{array}{l}\text { FJS-12 Q07 } \\
\text { Baseline - } \\
\text { FJS-12 Q07 } \\
\text { Follow-up }\end{array}$ & -0.29 & $-0.73-0.15$ & $-0.63-0.04$ & 0.61 & $0.29-0.84$ & $0.24-0.84$ & 0.18 & 0.20 & 0.18 \\
\hline $\begin{array}{l}\text { FJS-12 Q08 } \\
\text { Baseline - } \\
\text { FJS-12 Q08 } \\
\text { Follow-up }\end{array}$ & -0.38 & $-0.82-0.07$ & $-0.79-0.00$ & 0.54 & $0.05-0.89$ & $-0.02-0.90$ & 0.10 & 0.14 & 0.13 \\
\hline $\begin{array}{l}\text { FJS-12 Q09 } \\
\text { Baseline - } \\
\text { FJS-12 Q09 } \\
\text { Follow-up }\end{array}$ & -0.08 & $-0.41-0.24$ & $-0.33-0.21$ & 0.72 & $0.37-0.87$ & $0.33-0.89$ & 0.60 & 0.61 & 0.59 \\
\hline $\begin{array}{l}\text { FJS-12 Q10 } \\
\text { Baseline - } \\
\text { FJS-12 Q10 } \\
\text { Follow-up }\end{array}$ & 0.04 & $-0.45-0.53$ & $-0.38-0.46$ & 0.44 & $0.00-0.82$ & $-0.05-0.84$ & 0.86 & 0.88 & 0.86 \\
\hline $\begin{array}{l}\text { FJS-12 Q11 } \\
\text { Baseline - } \\
\text { FJS-12 Q11 } \\
\text { Follow-up }\end{array}$ & -0.17 & $-0.46-0.13$ & $-0.42-0.04$ & 0.80 & $0.53-0.92$ & $0.56-0.90$ & 0.26 & 0.28 & 0.27 \\
\hline $\begin{array}{l}\text { FJS-12 Q12 } \\
\text { Baseline - } \\
\text { FJS-12 Q12 } \\
\text { Follow-up }\end{array}$ & -0.21 & $-0.62-0.20$ & $-0.56-0.13$ & 0.66 & $0.32-0.84$ & $0.37-0.82$ & 0.31 & 0.32 & 0.30 \\
\hline $\begin{array}{l}\text { FJS-12 Total } \\
\text { Baseline - } \\
\text { FJS-12 Total } \\
\text { Follow-up }\end{array}$ & 3.26 & $-3.24-10.36$ & $-2.64-10.02$ & 0.79 & $0.58-0.92$ & $0.60-0.88$ & 0.29 & 0.31 & 0.27 \\
\hline $\begin{array}{l}\text { OKS Q01 } \\
\text { Baseline - } \\
\text { OKS Q01 } \\
\text { Follow-up }\end{array}$ & 0.04 & $-0.11-0.19$ & $-0.14-0.21$ & 0.94 & $0.83-0.99$ & $0.85-0.98$ & 0.66 & 0.69 & 0.66 \\
\hline
\end{tabular}


Table 9 (continued)

\begin{tabular}{|c|c|c|c|c|c|c|c|c|c|}
\hline \multirow{2}{*}{$\begin{array}{l}\text { Question } \\
\text { and } \\
\text { timepoint }\end{array}$} & \multirow[b]{2}{*}{$\begin{array}{l}\text { Mean } \\
\text { difference }\end{array}$} & \multirow{2}{*}{$\begin{array}{l}\text { Bootstrapping } \\
N=200 \\
95 \% \mathrm{Cl} \text { of mean } \\
\text { difference after } \\
\text { bootstrap }\end{array}$} & \multirow{2}{*}{$\begin{array}{l}\text { Bootstrapping } \\
N=1000 \\
95 \% \mathrm{Cl} \text { of mean } \\
\text { difference after } \\
\text { bootstrap }\end{array}$} & \multirow[b]{2}{*}{$\begin{array}{l}\text { Correlation } \\
\text { coefficient }\end{array}$} & \multirow{2}{*}{$\begin{array}{l}\text { Bootstrapping } \\
N=200 \\
95 \% \mathrm{Cl} \text { of } \\
\text { correlation } \\
\text { coefficient after } \\
\text { bootstrap }\end{array}$} & \multirow{2}{*}{$\begin{array}{l}\text { Bootstrapping } \\
N=1000 \\
95 \% \mathrm{Cl} \text { of } \\
\text { correlation } \\
\text { coefficient after } \\
\text { bootstrap }\end{array}$} & \multirow[b]{2}{*}{$P$ value } & \multirow{2}{*}{$\begin{array}{l}\text { Bootstrapping } \\
N=200 \\
P \text { value after } \\
\text { bootstrap }\end{array}$} & \multirow{2}{*}{$\begin{array}{l}\text { Bootstrapping } \\
N=1000 \\
P \text { value after } \\
\text { bootstrap }\end{array}$} \\
\hline & & & & & & & & & \\
\hline $\begin{array}{l}\text { OKS Q02 } \\
\text { Baseline - } \\
\text { OKS Q02 } \\
\text { Follow-up }\end{array}$ & 0.07 & $-0.19-0.41$ & $-0.22-0.36$ & 0.66 & $0.36-0.81$ & $0.46-0.82$ & 0.60 & 0.57 & 0.59 \\
\hline $\begin{array}{l}\text { OKS Q03 } \\
\text { Baseline - } \\
\text { OKS Q03 } \\
\text { Follow-up }\end{array}$ & 0.04 & $-0.30-0.26$ & $-0.24-0.32$ & 0.71 & $0.51-0.86$ & $0.45-0.90$ & 0.79 & 0.82 & 0.79 \\
\hline $\begin{array}{l}\text { OKS Q04 } \\
\text { Baseline - } \\
\text { OKS Q04 } \\
\text { Follow-up }\end{array}$ & 0.07 & $-0.22-0.41$ & $-0.32-0.47$ & 0.34 & $-0.05-0.68$ & $-0.05-0.68$ & 0.70 & 0.67 & 0.70 \\
\hline $\begin{array}{l}\text { OKS Q05 } \\
\text { Baseline - } \\
\text { OKS Q05 } \\
\text { Follow-up }\end{array}$ & 0.11 & $-0.07-0.33$ & $-0.17-0.39$ & 0.68 & $0.42-0.88$ & $0.40-0.90$ & 0.42 & 0.40 & 0.41 \\
\hline $\begin{array}{l}\text { OKS Q06 } \\
\text { Baseline - } \\
\text { OKS Q06 } \\
\text { Follow-up }\end{array}$ & 0.04 & $-0.19-0.30$ & $-0.20-0.27$ & 0.89 & $0.77-0.94$ & $0.77-0.95$ & 0.75 & 0.69 & 0.78 \\
\hline $\begin{array}{l}\text { OKS Q07 } \\
\text { Baseline - } \\
\text { OKS Q07 } \\
\text { Follow-up }\end{array}$ & 0.15 & $-0.24-0.56$ & $-0.33-0.62$ & 0.65 & $0.36-0.87$ & $0.35-0.85$ & 0.53 & 0.54 & 0.51 \\
\hline $\begin{array}{l}\text { OKS Q08 } \\
\text { Baseline - } \\
\text { OKS Q08 } \\
\text { Follow-up }\end{array}$ & 0.11 & $-0.16-0.37$ & $-0.19-0.41$ & 0.79 & $0.64-0.89$ & $0.54-0.93$ & 0.45 & 0.46 & 0.46 \\
\hline $\begin{array}{l}\text { OKS Q09 } \\
\text { Baseline - } \\
\text { OKS Q09 } \\
\text { Follow-up }\end{array}$ & 0.00 & $-0.22-0.27$ & $-0.31-0.31$ & 0.66 & $0.41-0.89$ & $0.39-0.89$ & 1.00 & 1.00 & 1.00 \\
\hline $\begin{array}{l}\text { OKS Q10 } \\
\text { Baseline - } \\
\text { OKS Q10 } \\
\text { Follow-up }\end{array}$ & -0.07 & $-0.33-0.26$ & $-0.38-0.24$ & 0.64 & $0.38-0.82$ & $0.37-0.83$ & 0.63 & 0.63 & 0.62 \\
\hline $\begin{array}{l}\text { OKS Q11 } \\
\text { Baseline - } \\
\text { OKS Q11 } \\
\text { Follow-up }\end{array}$ & 0.07 & $-0.17-0.33$ & $-0.24-0.38$ & 0.77 & $0.56-0.89$ & $0.54-0.91$ & 0.63 & 0.61 & 0.64 \\
\hline $\begin{array}{l}\text { OKS Q12 } \\
\text { Baseline - } \\
\text { OKS Q12 } \\
\text { Follow-up }\end{array}$ & 0.41 & $0.07-0.75$ & $0.01-0.81$ & 0.62 & $0.40-0.81$ & $0.41-0.84$ & 0.05 & 0.02 & 0.05 \\
\hline $\begin{array}{l}\text { OKS Total } \\
\text { Baseline - } \\
\text { OKS Total } \\
\text { Follow-up }\end{array}$ & -4.22 & $-10.70-2.31$ & $-11.80-3.35$ & -0.91 & $-0.96-0.80$ & $-0.97--0.79$ & 0.26 & 0.24 & 0.27 \\
\hline
\end{tabular}

important to provide accurate and high responsiveness to the respondents (patients) who are "overweight" or "obese". The effect of BMI on results from different PRO questionnaires are somehow conflicting [51-53]. FJS-12 has been proven to be simple, valid and reliable in original and translated versions [3, 17, 20, 54-56]. A study in New York found that although patients who were obese
$\left(\mathrm{BMI} \geq 30 \mathrm{~kg} / \mathrm{m}^{2}\right)$ and received primary TKA provided lower post-surgery FJS-12 scores, statistical significance was not found [57]. That means FJS-12 is able to accurately reflect patients' outcome undergoing conservative or operative treatment of the knee, regardless of the patient's BMI. The mean BMI of our patients was 27.48 which was classified as "obese" (using BMI categories 
Table 10 Summary table of the $95 \% \mathrm{Cl}$ of mean difference, correlation coefficient, and $p$ value comparing the scores in individual questions and total between FJS-12 and OKS at baseline using paired T-tests applying bootstrapping with $N=200$ and $N=1000$

\begin{tabular}{|c|c|c|c|c|c|c|c|c|c|}
\hline \multirow{2}{*}{$\begin{array}{l}\text { Question } \\
\text { and } \\
\text { timepoint }\end{array}$} & \multirow[b]{2}{*}{$\begin{array}{l}\text { Mean } \\
\text { difference }\end{array}$} & \multirow{2}{*}{$\begin{array}{l}\text { Bootstrapping } \\
N=200 \\
95 \% \mathrm{Cl} \text { of mean } \\
\text { difference after } \\
\text { bootstrap }\end{array}$} & \multirow{2}{*}{$\begin{array}{l}\text { Bootstrapping } \\
N=1000 \\
95 \% \mathrm{Cl} \text { of mean } \\
\text { difference after } \\
\text { bootstrap }\end{array}$} & \multirow[b]{2}{*}{$\begin{array}{l}\text { Correlation } \\
\text { coefficient }\end{array}$} & \multirow{2}{*}{$\begin{array}{l}\text { Bootstrapping } \\
N=200 \\
95 \% \mathrm{Cl} \text { of } \\
\text { correlation } \\
\text { coefficient after } \\
\text { bootstrap }\end{array}$} & \multirow{2}{*}{$\begin{array}{l}\text { Bootstrapping } \\
N=1000 \\
95 \% \mathrm{Cl} \text { of } \\
\text { correlation } \\
\text { coefficient after } \\
\text { bootstrap }\end{array}$} & \multirow[b]{2}{*}{$P$ value } & \multirow{2}{*}{$\begin{array}{l}\text { Bootstrapping } \\
N=200 \\
P \text { value after } \\
\text { bootstrap }\end{array}$} & \multirow{2}{*}{$\begin{array}{l}\text { Bootstrapping } \\
N=1000 \\
P \text { value after } \\
\text { bootstrap }\end{array}$} \\
\hline & & & & & & & & & \\
\hline $\begin{array}{l}\text { FJS-12 Q01 } \\
\text { Baseline - } \\
\text { OKS Q01 } \\
\text { Baseline }\end{array}$ & -0.21 & $-0.46-0.00$ & $-0.42-0.00$ & 0.87 & $0.73-0.95$ & $0.71-0.94$ & 0.10 & 0.10 & 0.09 \\
\hline $\begin{array}{l}\text { FJS-12 Q02 } \\
\text { Baseline - } \\
\text { OKS Q02 } \\
\text { Baseline }\end{array}$ & 0.08 & $-0.19-0.36$ & $-0.13-0.29$ & 0.86 & $0.73-0.93$ & $0.70-0.94$ & 0.54 & 0.51 & 0.52 \\
\hline $\begin{array}{l}\text { FJS-12 Q03 } \\
\text { Baseline - } \\
\text { OKS Q03 } \\
\text { Baseline }\end{array}$ & 0.17 & $-0.34-0.68$ & $-0.25-0.58$ & 0.62 & $0.26-0.87$ & $0.28-0.83$ & 0.50 & 0.48 & 0.49 \\
\hline $\begin{array}{l}\text { FJS-12 Q04 } \\
\text { Baseline - } \\
\text { OKS Q04 } \\
\text { Baseline }\end{array}$ & -0.38 & $-0.90-0.15$ & $-0.83-0.00$ & 0.64 & $0.34-0.86$ & $0.25-0.86$ & 0.15 & 0.17 & 0.16 \\
\hline $\begin{array}{l}\text { FJS-12 Q05 } \\
\text { Baseline - } \\
\text { OKS Q05 } \\
\text { Baseline }\end{array}$ & -0.25 & $-0.88-0.38$ & $-0.83-0.29$ & 0.36 & $-0.11-0.72$ & $-0.08-0.74$ & 0.42 & 0.41 & 0.42 \\
\hline $\begin{array}{l}\text { FJS-12 Q06 } \\
\text { Baseline - } \\
\text { OKS Q06 } \\
\text { Baseline }\end{array}$ & -0.04 & $-0.46-0.38$ & $-0.46-0.33$ & 0.60 & $0.00-0.95$ & $-0.12-0.96$ & 0.84 & 0.88 & 0.85 \\
\hline $\begin{array}{l}\text { FJS-12 Q07 } \\
\text { Baseline - } \\
\text { OKS Q07 } \\
\text { Baseline }\end{array}$ & -0.29 & $-0.73-0.15$ & $-0.63-0.04$ & 0.61 & $0.29-0.84$ & $0.24-0.84$ & 0.18 & 0.20 & 0.18 \\
\hline $\begin{array}{l}\text { FJS-12 Q08 } \\
\text { Baseline - } \\
\text { OKS Q08 } \\
\text { Baseline }\end{array}$ & -0.38 & $-0.82-0.07$ & $-0.79-0.00$ & 0.54 & $0.05-0.89$ & $-0.02-0.90$ & 0.10 & 0.14 & 0.13 \\
\hline $\begin{array}{l}\text { FJS-12 Q09 } \\
\text { Baseline - } \\
\text { OKS Q09 } \\
\text { Baseline }\end{array}$ & -0.08 & $-0.41-0.24$ & $-0.33-0.21$ & 0.72 & $0.37-0.87$ & $0.33-0.89$ & 0.60 & 0.61 & 0.59 \\
\hline $\begin{array}{l}\text { FJS-12 Q10 } \\
\text { Baseline - } \\
\text { OKS Q10 } \\
\text { Baseline }\end{array}$ & 0.04 & $-0.45-0.53$ & $-0.38-0.46$ & 0.44 & $0.00-0.82$ & $-0.05-0.84$ & 0.86 & 0.88 & 0.86 \\
\hline $\begin{array}{l}\text { FJS-12 Q11 } \\
\text { Baseline - } \\
\text { OKS Q111 } \\
\text { Baseline }\end{array}$ & -0.17 & $-0.46-0.13$ & $-0.42-0.04$ & 0.80 & $0.53-0.92$ & $0.56-0.90$ & 0.26 & 0.28 & 0.27 \\
\hline $\begin{array}{l}\text { FJS-12 Q12 } \\
\text { Baseline - } \\
\text { OKS Q12 } \\
\text { Baseline }\end{array}$ & -0.21 & $-0.62-0.20$ & $-0.56-0.13$ & 0.66 & $0.32-0.84$ & $0.37-0.82$ & 0.31 & 0.32 & 0.30 \\
\hline $\begin{array}{l}\text { FJS-12 Total } \\
\text { Baseline - } \\
\text { OKS Total } \\
\text { Baseline }\end{array}$ & 3.26 & $-3.24-10.36$ & $-2.64-10.02$ & 0.79 & $0.58-0.92$ & $0.60-0.88$ & 0.29 & 0.31 & 0.27 \\
\hline
\end{tabular}


Table 11 Summary table of the $95 \% \mathrm{Cl}$ of mean difference, correlation coefficient, and $p$ value comparing the scores in individual questions and total between FJS-12 and OKS at follow-up using paired T-tests applying bootstrapping with $N=200$ and $N=1000$

\begin{tabular}{|c|c|c|c|c|c|c|c|c|c|}
\hline \multirow{2}{*}{$\begin{array}{l}\text { Question } \\
\text { and } \\
\text { timepoint }\end{array}$} & \multirow[b]{2}{*}{$\begin{array}{l}\text { Mean } \\
\text { difference }\end{array}$} & \multirow{2}{*}{$\begin{array}{l}\text { Bootstrapping } \\
N=200 \\
95 \% \mathrm{Cl} \text { of mean } \\
\text { difference after } \\
\text { bootstrap }\end{array}$} & \multirow{2}{*}{$\begin{array}{l}\text { Bootstrapping } \\
N=1000 \\
95 \% \mathrm{Cl} \text { of mean } \\
\text { difference after } \\
\text { bootstrap }\end{array}$} & \multirow[b]{2}{*}{$\begin{array}{l}\text { Correlation } \\
\text { coefficient }\end{array}$} & \multirow{2}{*}{$\begin{array}{l}\text { Bootstrapping } \\
N=200 \\
95 \% \mathrm{Cl} \text { of } \\
\text { correlation } \\
\text { coefficient after } \\
\text { bootstrap }\end{array}$} & \multirow{2}{*}{$\begin{array}{l}\text { Bootstrapping } \\
N=1000 \\
95 \% \mathrm{Cl} \text { of } \\
\text { correlation } \\
\text { coefficient after } \\
\text { bootstrap }\end{array}$} & \multirow[b]{2}{*}{$P$ value } & \multirow{2}{*}{$\begin{array}{l}\text { Bootstrapping } \\
N=200 \\
P \text { value after } \\
\text { bootstrap }\end{array}$} & \multirow{2}{*}{$\begin{array}{l}\text { Bootstrapping } \\
N=1000 \\
P \text { value after } \\
\text { bootstrap }\end{array}$} \\
\hline & & & & & & & & & \\
\hline $\begin{array}{l}\text { FJS-12 Q01 } \\
\text { Follow-up } \\
\text { - OKS Q01 } \\
\text { Follow-up }\end{array}$ & 1.29 & $0.43-2.14$ & $0.43-2.14$ & -0.78 & $-0.87--0.65$ & $-0.88-0.64$ & 0.01 & 0.02 & 0.01 \\
\hline $\begin{array}{l}\text { FJS-12 Q02 } \\
\text { Follow-up } \\
\text { - OKS Q02 } \\
\text { Follow-up }\end{array}$ & 0.18 & $-0.55-0.91$ & $-0.55-0.91$ & -0.53 & $-0.79--0.29$ & $-0.81--0.26$ & 0.62 & 0.68 & 0.62 \\
\hline $\begin{array}{l}\text { FJS-12 Q03 } \\
\text { Follow-up } \\
\text { - OKS Q03 } \\
\text { Follow-up }\end{array}$ & 0.32 & $-0.48-1.12$ & $-0.48-1.12$ & -0.57 & $-0.80--0.27$ & $-0.79--0.25$ & 0.42 & 0.44 & 0.42 \\
\hline $\begin{array}{l}\text { FJS-12 Q04 } \\
\text { Follow-up } \\
\text { - OKS Q04 } \\
\text { Follow-up }\end{array}$ & -0.29 & $-1.03-0.46$ & $-1.03-0.46$ & -0.39 & $-0.70-0.03$ & $-0.67--0.08$ & 0.44 & 0.48 & 0.44 \\
\hline $\begin{array}{l}\text { FJS-12 Q05 } \\
\text { Follow-up } \\
\text { - OKS Q05 } \\
\text { Follow-up }\end{array}$ & 0.54 & $-0.21-1.28$ & $-0.21-1.28$ & -0.65 & $-0.85--0.37$ & $-0.84--0.39$ & 0.15 & 0.17 & 0.15 \\
\hline $\begin{array}{l}\text { FJS-12 Q06 } \\
\text { Follow-up } \\
\text { - OKS Q06 } \\
\text { Follow-up }\end{array}$ & 1.21 & $0.47-1.96$ & $0.47-1.96$ & -0.53 & $-0.75--0.28$ & $-0.73--0.30$ & $<0.01$ & 0.01 & $<0.01$ \\
\hline $\begin{array}{l}\text { FJS-12 Q07 } \\
\text { Follow-up } \\
\text { - OKS Q07 } \\
\text { Follow-up }\end{array}$ & 2.39 & $1.52-3.27$ & $1.52-3.27$ & -0.50 & $-0.81-0.01$ & $-0.83-0.02$ & $<0.01$ & 0.01 & $<0.01$ \\
\hline $\begin{array}{l}\text { FJS-12 Q08 } \\
\text { Follow-up } \\
\text { - OKS Q08 } \\
\text { Follow-up }\end{array}$ & 1.46 & $0.79-2.14$ & $0.79-2.14$ & -0.35 & $-0.60--0.06$ & $-0.56--0.05$ & $<0.01$ & 0.01 & $<0.01$ \\
\hline $\begin{array}{l}\text { FJS-12 Q09 } \\
\text { Follow-up } \\
\text { - OKS Q09 } \\
\text { Follow-up }\end{array}$ & 1.36 & $0.69-2.03$ & $0.69-2.03$ & -0.46 & $-0.67--0.27$ & $-0.63--0.31$ & $<0.01$ & 0.01 & $<0.01$ \\
\hline $\begin{array}{l}\text { FJS-12 Q10 } \\
\text { Follow-up } \\
\text { - OKS Q10 } \\
\text { Follow-up }\end{array}$ & 0.57 & $-0.09-1.23$ & $-0.09-1.23$ & -0.47 & $-0.69--0.21$ & $-0.69--0.22$ & 0.09 & 0.10 & 0.09 \\
\hline $\begin{array}{l}\text { FJS-12 Q11 } \\
\text { Follow-up } \\
\text { - OKS Q11 } \\
\text { Follow-up }\end{array}$ & 0.54 & $-0.25-1.32$ & $-0.25-1.32$ & -0.67 & $-0.82--0.47$ & $-0.83--0.49$ & 0.17 & 0.19 & 0.17 \\
\hline $\begin{array}{l}\text { FJS-12 Q12 } \\
\text { Follow-up } \\
\text { - OKS Q12 } \\
\text { Follow-up }\end{array}$ & 1.04 & $0.29-1.78$ & $0.29-1.78$ & -0.27 & $-0.58--0.24$ & $-0.57-0.07$ & 0.01 & 0.01 & 0.01 \\
\hline $\begin{array}{l}\text { FJS-12 Total } \\
\text { Follow-up } \\
\text { - OKS Total } \\
\text { Follow-up }\end{array}$ & 7.21 & $1.38-13.04$ & $1.38-13.04$ & -0.88 & $0.80-0.94$ & $0.79-0.95$ & 0.02 & 0.03 & 0.02 \\
\hline
\end{tabular}


for Asians [58]). We speculate the percentage of obese patients would be ever increasing. The education level of our patients also reflects the necessity of having a Traditional Chinese-Hong Kong version of FJS-12 for local community. The validated FJS-12 is, therefore, suitable for any patients who linguistically prefer Traditional ChineseHong Kong version.

There are 3 questions which either ICC or Cronbach's alpha was lower than 0.7. The 3 questions are: Q3. when you are walking for more than $15 \mathrm{~min}, \mathrm{Q} 8$. when you are standing up from a low-sitting position, and Q10. when you are doing housework or gardening. Looking at the percentages of "floor" and "ceiling" answers in these questions can identify the causes. In question 3, 24.7\% of patients were never aware of their artificial joints when walking for more than $15 \mathrm{~min}$ (the higher percentage of "never" means better (already forgotten their artificial joints)) and this percentage has been decreased to $17.9 \%$ after 2 weeks. Similarly, the percentage of answering "mostly" increased by $4.2 \%(17.9 \%-13.7 \%)$ meaning more patients took extra attention to their knee implants after at least 15-min walk. Patients tended not to "forget their knee implants" within the test-retest period. In this study, the period administering both questionnaires between the 2 rounds was about 2 weeks, which was similar in other validation studies $[25,55,59]$. As a result, the percentages had been changed and the changes made the ICC and Cronbach's alpha lower comparing with other questions. Similar phenomenon was also observed in question 10 (patients were "alerted" and fewer patients forgot their knee implants when doing housework). In question 8 , statistical significances were found between patients scoring "mostly" and "never" in both baseline and follow-up. Percentages of patients reflecting "never" thought of their artificial knee joints increased from $32.0 \%$ at baseline to $42.9 \%$ at follow-up, and at the same time, the percentages of patients mostly aware of their knees decreased. The results of Q8 (when you are standing up from a low-sitting position) are contrary to those of Q3 (when you are walking for more than $15 \mathrm{~min}$ ) and Q10 (when you are doing housework or gardening) because walking for more than $15 \mathrm{~min}$ and doing housework or gardening are continuously performing while standing up from a low-sitting position is an example of split-second movement. Patients on artificial knee joints tend to be aware of their joints after these kinds of continuous activities over time (reflected by the decreased ceiling percentages and increased floor percentages). Patients gain confidence on short-term movements over time; therefore, more patients "forget" their artificial joint(s) when they stand up from a lower-sitting position. No significant floor and ceiling effect was observed through a recent validity study in the UK evaluating the Oxford Knee Score using a national patient-reported outcome measure dataset [60].

Correlations between translated FJS-12 and OKS are promising. We correlated FJS-12 with OKS at baseline and follow-up, and results were 0.598 at baseline and 0.879 at follow-up. In different validation studies on language adaptations using OKS as gold standard, correlation coefficients were 0.366 in German version [55] and 0.37 in Hindi version [59]. Our results showed moderate correlation when patients first answered the FJS-12 and good correlation at the repeated administration. Previous studies showed FJS-12 was more responsive at 6 months and 12 months[61], and 1 to 2 years after surgery [18]. We conclude that the responsiveness of FJS-12 is good for knee OA patients after TKA. The subjects in this study experienced TKA at least after 1 year and the responsiveness of FJS-12 was proven better 1 to 2 years after surgery [18]. Further study on inviting patients to complete FJS-12 shortly after TKA to look for the responsiveness immediately after surgery to 6 or 9 months after can fill out responsiveness data gap before 1 year after TKA. We chose OKS as the gold standard because both questionnaires share similar construct (12 questions) and total sum is calculated by simply adding all 12 scores ("final score" in FJS-12 and "overall score" in OKS; data conversion reverting the score strength in FJS-12 is require without data transformation). Both total sums can be scaled to a maximum of 100 (native in FJS-12 and ratio conversion in OKS). That would make the two questionnaires easily comparable. Furthermore, only OKS is introduced in this study which is different from other studies which employed multiple PRO tools to validate the language adapted version. The mean age of our patients was around 70 years old [49] and response bias happened when old age patients required to fill out multiple questionnaires. Telephone interview instead of face-to-face interview could have been an alternative but declined eventually because the targets were elderly patients who were prone to lower response rates [62-64] and they could cope with short interview duration only $[62,63]$. Mailing all sets of questionnaires to the participants hoping them to complete and send the questionnaires back at different time points was reported low response rate. The Dutch version came with a limitation of receiving all questionnaires back after sending two sets of questionnaires in one go expecting to receive the second set within 2 weeks [65]. Further study on developing an electronic version of FJS-12 and accessing the FJS-12 through a web/ mobile browser or mobile phone application could possibly increase the successful rate. Furthermore, if the 
electronic version can easily switch languages instantly, that will definitely increase the response rate in communities which use different kinds of official languages e.g., switch between English or French in Canada.

Using Bland-Altman (BA) plot explaining the agreement between two methods or test-retest reliability is very useful and clear to demonstrate any systematic error between the two measures. This confirms the good testretest (baseline-follow-up) agreement and reproducibility of FJS-12. Our previous experience on the use of BA plots to evaluate the agreements between a new imaging technology to the conventional X-ray methods was proven useful [66].

Another important message we would like to bring out from this study is to raise the awareness of subculture difference within the same ethnicity or race. We firstly introduce this point by referencing to the experience of cultural adaptation and validation of WHOQOL questionnaire. WHOQOL had been translated to Chinese (China), Chinese (Hong Kong), and Chinese (Taiwan) languages [24]. Later, the Taiwan Chinese language adaption group published another article on testing the agreement between "Taiwan Chinese" version and "Taiwanese" version of the brief version of the WHOQOL [67]. The authors pointed out that $>50 \%$ of the elderly Taiwanese at age over 65 only used a spoken language, Taiwanese. Another classic example we mentioned before, is that WHOQOL is also available in American English, Canadian English, British English, and Australian English. We speculate that sub-culture variations happen in African countries, European countries, middle East countries, Southeast Asia countries, and possibly any countries with multicultural societies or federal multicultural policies. In summary, sub-culture difference is recommended to review and consider including in future version of IQOLA project. Further longitudinal study examining the long-term reflection of FJS-12 scores to patients underwent TKA is also recommended to look for any practical change over time.

\section{Limitations of this study}

The small sample size in this study reduces the data generalizability and affects the accuracy and reliability of the results of this study. This study was carried out during COVID-19 pandemic and the patients were recruited when the local situation was being eased. We stuck onto the original research protocol to collect two sets of questionnaires through face-to-face interview. Moreover, we introduced bootstrapping to tackle the small sample size issue. Bootstrapping is an appropriate way to control and check the stability of the result. The estimates of standard errors and confidence intervals are both promising after bootstrapping for $N=200$ and $N=1000$. Second, we admit that using multiple gold standards increase the validity of the translated version. However, our experience tells us that when patients move on to the second questionnaire, they start asking questions on why the questions are similar to the first one. Some patients requested to opt out from the study. This affects the compliance rate. Therefore, we choose the well-recognized patient subjective outcome assessment (i.e., OKS) as the sole gold standard in this study. In view of this situation, NRS was added to correlate with FJS-12 although NRS might not be classified as "gold standard tool". Minimal important change (MIC) in calculating responsiveness is an estimate which needs to establish a gold standard. MIC of Hindu version is 8.67 and 10.9 in German version. Further study on standardizing the calculation of MIC is recommended.

\section{Conclusions}

Traditional Chinese-Hong Kong version of FJS-12 showed good reliability and validity for patients underwent TKA. The "Forgotten joint" score questionnaire did a great job to evaluate how the patients "forget" their artificial joint during their daily activities. FJS-12 is also suitable for patients who are obese (or body mass index (BMI) non-specific). Individual questions and final score did not carry any floor effect and ceiling effect. FJS-12 also found to have good agreement, nice responsiveness and discriminant validity. FJS-12 are important PRO questionnaires for patients who come across TKA with benefits outstand other PRO tools. Moreover, subcultural adaptation should be considered along with the standard guideline during cross-cultural adaptation and validation.

\section{Abbreviations}

FJS-12: Forgotten Joint Score-12; PRO: Patient-reported outcome; HRQOL: Health-related quality of life; WOMAC: Western Ontario and McMaster Universities Osteoarthritis Index; OKS: Oxford Knee Score; NRS: Numeric Rating Scale; KOOS: Knee Injury and Osteoarthritis Outcome Score; KSS: Knee Society Score; KFS: Knee Function Score; TKA: Total knee arthroplasty; FJS-12: Chinese Cantonese (Hong Kong) version of Forgotten Joint Score-12; ISPOR: The Professional Society for Health Economics and Outcomes Research; SD: Standard deviation; ICC: Intra-class correlation; SDC: Smallest detectable change; MIC: Minimal important change; LOA: Limits of agreement; BMI: Body mass index; OA: Osteoarthritis; WHO: World Health Organization; BAplot: Bland-Altmanplot.

\section{Supplementary Information}

The online version contains supplementary material available at https://doi. org/10.1186/s12891-022-05156-5.

Additional file1 


\section{Authors' contributions}

LCML, MTYO, and KKWH designed the research, collected the data. WWC and KKWH wrote the paper. WWC assembled the data. WWC analysed, interpreted the data and wrote the paper. All authors took part in the writing and final editing of the manuscript. All authors have been given a copy of the manuscript, all have approved the final version of the manuscript, and all are prepared to take public responsibility for the work and share responsibility and accountability for the results.

\section{Funding}

This research did not receive any specific grant from funding agencies in the public, commercial, or not-for-profit sectors.

\section{Availability of data and material}

The datasets used and/or analysed during the current study are available from the corresponding author on reasonable request.

\section{Declarations}

\section{Ethics approval and consent to participate}

Ethical approval was obtained from the ethics review board of the Joint NTEC/CUHK Ethics Committee (Research Ethics Committee approval number: 2019.337).The study was performed in accordance with the Declaration of Helsinki and ICH-GCP.Written informed consent was obtained from all participants

\section{Consent for publication}

Not applicable.

\section{Competing interests}

The authors declare that they have no competing interests.

\section{Author details}

${ }^{1}$ Department of Orthopaedics and Traumatology, Chinese University of Hong Kong, Hong Kong SAR, China. ${ }^{2}$ Department of Orthopaedics and Traumatology, Prince of Wales Hospital, Hong Kong SAR, China.

\section{Received: 13 May 2021 Accepted: 25 February 2022}

Published online: 08 March 2022

\section{References}

1. Guyatt GH, Feeny DH, Patrick DL. Measuring health-related quality of life Ann Intern Med. 1993;118(8):622-9.

2. Guillemin F, Bombardier C, Beaton D. Cross-cultural adaptation of health-related quality of life measures: literature review and proposed guidelines. J Clin Epidemiol. 1993;46(12):1417-32.

3. Behrend $\mathrm{H}$, Giesinger K, Giesinger JM, Kuster MS. The "Forgotten Joint" as the Ultimate Goal in Joint Arthroplasty: Validation of a New PatientReported Outcome Measure. J Arthroplasty. 2012:27(3):430-436.e431.

4. Deroche E, Batailler C, Swan J, Sappey-Marinier E, Neyret P, Servien E, Lustig S: No difference between resurfaced and non-resurfaced patellae with a modern prosthesis design: a prospective randomized study of 250 total knee arthroplasties. Knee Surgery, Sports Traumatology, Arthroscopy. 2021. Mar 4. Online ahead of print.

5. Howell SM, Gill M, Shelton TJ, Nedopil AJ: Reoperations are few and confined to the most valgus phenotypes 4 years after unrestricted calipered kinematically aligned TKA. Knee Surgery, Sports Traumatology, Arthroscopy. 2021. Feb 13. Epub ahead of print.

6. Domb BG, Chen JW, Kyin C, Bheem R, Karom J, Shapira J, Rosinsky PJ, Lall AC, Maldonado DR. Primary Robotic-Arm Assisted Total Hip Arthroplasty: An Analysis of 501 Hips With 44-Month Follow-up. Orthopedics. 2021:44(2):70-6.

7. Putman S, Dartus J, Migaud H, Pasquier G, Girard J, Preda C, Duhamel A. A: Can the minimal clinically important difference be determined in a French-speaking population with primary hip replacement using one PROM item and the Anchor strategy? Orthop Traumatol Surg Res. 2021:107(3):102830
8. Zambianchi F, Franceschi G, Banchelli F, Marcovigi A, Ensini A, Catani F: Robotic Arm-Assisted Lateral Unicompartmental Knee Arthroplasty: How Are Components Aligned? J Knee Surg. 2021. Jan 28. Epub ahead of print

9. Nakajima A, Yamada M, Sonobe M, Akatsu Y, Saito M, Yamamoto K, Saito J, Norimoto M, Koyama K, Takahashi H, et al. Three-year clinical and radiological results of a cruciate-retaining type of the knee prosthesis with anatomical geometry developed in Japan. BMC Musculoskelet Disord. 2021;22(1):241

10. Jie K, Feng W, Li F, Wu K, Chen J, Zhou G, Zeng H, Zeng Y. Long-term survival and clinical outcomes of non-vascularized autologous and allogeneic fibular grafts are comparable for treating osteonecrosis of the femoral head. J Orthop Surg Res. 2021;16(1):109.

11. Bellamy N, Buchanan WW. Outcome measurement in osteoarthritis clinical trials: The case for standardisation. Clin Rheumatol. 1984;3(3):293-303.

12. Bellamy N, Buchanan WW. A preliminary evaluation of the dimensionality and clinical importance of pain and disability in osteoarthritis of the hip and knee. Clin Rheumatol. 1986;5(2):231-41.

13. Murray DW, Fitzpatrick R, Rogers K, Pandit H, Beard DJ, Carr AJ, Dawson $J$. The use of the Oxford hip and knee scores. J Bone Joint Surg Br. 2007;89(8):1010-4.

14. Roos EM, Roos HP, Lohmander LS, Ekdahl C, Beynnon BD. Knee Injury and Osteoarthritis Outcome Score (KOOS)-development of a self-administered outcome measure. J Orthop Sports Phys Ther. 1998;28(2):88-96.

15. Insall JN, Dorr LD, Scott RD, Scott WN. Rationale of the Knee Society clinical rating system. Clin Orthop Relat Res. 1989;248:13-4.

16. Gill JR, Corbett JA, Wastnedge E, Nicolai P. Forgotten Joint Score: Comparison between total and unicondylar knee arthroplasty. Knee. 2021;29:26-32

17. HD F, LF L, GJ M, G K, MD J, PJT, SAHR W, HC R. Validation of the English language Forgotten Joint Score-12 as an outcome measure for total hip and knee arthroplasty in a British population. Bone Joint J. 2017;99-B(2):218-24.

18. Giesinger K, Hamilton DF, Jost B, Holzner B, Giesinger JM. Comparative responsiveness of outcome measures for total knee arthroplasty. Osteoarthritis Cartilage. 2014;22(2):184-9.

19. Chithartha K, Nair AS, Thilak J. A long-term cross-sectional study with modified forgotten joint score to assess the perception of artificial joint after total knee arthroplasty. SICOT-J. 2021;7:14-14.

20. Freigang V, Weber J, Mueller K, Pfeifer C, Worlicek M, Alt V, Baumann FM. Evaluation of joint awareness after acetabular fracture: Validation of the Forgotten Joint Score according to the COSMIN checklist protocol. World journal of orthopedics. 2021;12(2):69-81.

21. Carlson VR, Post ZD, Orozco FR, Davis DM, Lutz RW, Ong AC. When Does the Knee Feel Normal Again: A Cross-Sectional Study Assessing the Forgotten Joint Score in Patients After Total Knee Arthroplasty. J Arthroplasty. 2018;33(3):700-3.

22. Thomsen MG, Latifi R, Kallemose T, Barfod KW, Husted $H$, Troelsen A. Good validity and reliability of the forgotten joint score in evaluating the outcome of total knee arthroplasty. Acta Orthop. 2016:87(3):280-5.

23. Thienpont E, Opsomer G, Koninckx A, Houssiau F. Joint awareness in different types of knee arthroplasty evaluated with the Forgotten Joint score. J Arthroplasty. 2014;29(1):48-51.

24. Yao G. Wu C-h: Similarities and Differences Among the Taiwan, China, and Hong-Kong Versions of the WHOQOL Questionnaire. Soc Indic Res. 2009:91(1):79-98.

25. Cao S, Liu N, Han W, Zi Y, Peng F, Li L, Fu Q, Chen Y, Zheng W, Qian Q. Simplified Chinese version of the Forgotten Joint Score (FJS) for patients who underwent joint arthroplasty: cross-cultural adaptation and validation. J Orthop Surg Res. 2017;12(1):6-6.

26. The Forgotten Joint Score [http://www.forgotten-joint-score.info/langu ages/]

27. Liao P-S, Fu Y-C, Yi C-C. Perceived quality of life in Taiwan and Hong Kong: an intra-culture comparison. J Happiness Stud. 2005;6(1):43-67.

28. Beaton DE, Bombardier C, Guillemin F, Ferraz MB. Guidelines for the process of cross-cultural adaptation of self-report measures. Spine (Phila Pa 1976). 2000:25(24):3186-91.

29. Bullinger $M$, Alonso J, Apolone $G$, Leplège $A$, Sullivan $M$, Wood-Dauphinee S, Gandek B, Wagner A, Aaronson N, et al. Translating health status questionnaires and evaluating their quality: the IQOLA Project 
approach. International Quality of Life Assessment. J Clin Epidemiol. 1998;51(11):913-23.

30. Dunbar MJ, Robertsson O, Ryd L, Lidgren L. Appropriate questionnaires for knee arthroplasty. Results of a survey of 3600 patients from The Swedish Knee Arthroplasty Registry. J Bone Joint Surg Br. 2001;83(3):339-44.

31. Bin Sheeha B, Williams A, Johnson DS, Granat M, Bin Nasser A, Jones R. Responsiveness, Reliability, and Validity of Arabic Version of Oxford Knee Score for Total Knee Arthroplasty. JBJS. 2020;102(15).

32. Paravlic AH, Pisot S, Mitic P, Pisot R. Validation of the Oxford Knee Score and Lower Extremity Functional Score questionnaires for use in Slovenia. Arch Orthop Trauma Surg. 2020;140(10):1515-22.

33. Ngwayi JRM, Tan J, Liang N, Porter DE. Reliability and validity of 3 different Chinese versions of the Oxford knee score (OKS). Arthroplasty. 2020;2(1):31.

34. Lampropoulou S, Nowicky AV. Evaluation of the numeric rating scale for perception of effort during isometric elbow flexion exercise. Eur J Appl Physiol. 2012;112(3):1167-75.

35. Hsieh T-H, Lee JJ. Yu EW-R, Hu H-Y, Lin S-Y, Ho C-Y: Association between obesity and education level among the elderly in Taipei, Taiwan between 2013 and 2015: a cross-sectional study. Sci Rep. 2020;10(1):20285.

36. Mokkink LB, Terwee CB, Knol DL, Stratford PW, Alonso J, Patrick DL, Bouter LM, de Vet HC. Protocol of the COSMIN study: COnsensus-based Standards for the selection of health Measurement INstruments. BMC Med Res Methodol. 2006;6:2.

37. Terwee CB, Bot SDM, de Boer MR, van der Windt DAWM, Knol DL, Dekker J, Bouter LM, de Vet HCW. Quality criteria were proposed for measurement properties of health status questionnaires. J Clin Epidemiol. 2007;60(1):34-42.

38. Efron BTRJ. An introduction to the bootstrap. New York: Chapman \& Hall; 1993.

39. Efron B, Gong G. A leisurely look at the bootstrap, the jackknife, and crossvalidation. Am Stat. 1983;37(1):36-48.

40. DiCiccio TJ, Efron B. Bootstrap confidence intervals. Stat Sci. 1996;11(3):189-212.

41. Efron B, Tibshirani RJ. An introduction to the bootstrap. Boca Raton: CRC press; 1994

42. Banjanovic ES, Osborne JW. Confidence intervals for effect sizes: Applying bootstrap resampling. Pract Assess Res Eval. 2016;21(1):5

43. Koo TK, Li MY. A Guideline of Selecting and Reporting Intraclass Correlation Coefficients for Reliability Research. J Chiropr Med. 2016;15(2):155-63.

44. Norman GR, Wyrwich KW, Patrick DL. The mathematical relationship among different forms of responsiveness coefficients. Qual Life Res. 2007;16(5):815-22.

45. Global Strategy on Diet, Physical Activity and Health - 2004 [https://www. who.int/publications/i/item/9241592222]

46. Global action plan on physical activity 2018-2030: more active people for a healthier world [https://www.who.int/ncds/prevention/physical-activ ity/global-action-plan-2018-2030/en/]

47. Kelly T, Yang W, Chen CS, Reynolds K, He J. Global burden of obesity in 2005 and projections to 2030. Int J Obes. 2008:32(9):1431-7.

48. Man SL-C, Chau W-W, Chung K-Y, Ho KKW. Hypoalbuminemia and obesity class II are reliable predictors of peri-prosthetic joint infection in patient undergoing elective total knee arthroplasty. Knee Surgery \& Related Research. 2020;32(1):21.

49. Ho KK-W, Lau LC-M, Chau W-W, Poon Q, Chung K-Y, Wong RM-Y. Endstage knee osteoarthritis with and without sarcopenia and the effect of knee arthroplasty - a prospective cohort study. BMC Geriatrics. 2021;21(1):2.

50. Li MM-L, Kwok JY-Y, Chung K-Y, CheungChiu K-WK-H, Chau W-W, Ho KK-W. Prospective randomized trial comparing efficacy and safety of intravenous and intra-articular tranexamic acid in total knee arthroplasty. Knee Surgery \& Related Research. 2020;32(1):62.

51. Y CJ, N LN, C CH, R BARH, N PH, TDK J, L CSYS. The influence of body mass index on functional outcome and quality of life after total knee arthroplasty. The Bone \& Joint Journal. 2016;98 B(6):780-5.

52. Järvenpää J, Kettunen J, Soininvaara T, Miettinen H, Kröger H. Obesity Has a Negative Impact on Clinical Outcome after Total Knee Arthroplasty. Scandinavian Journal of Surgery. 2012;101(3):198-203.
53. Baker P, Petheram T, Jameson S, Reed M, Gregg P, Deehan D. The Association Between Body Mass Index and the Outcomes of Total Knee Arthroplasty. JBJS. 2012;94(16):1501-8.

54. Matsumoto M, Baba T, Homma Y, Kobayashi H, Ochi H, Yuasa T, Behrend $\mathrm{H}$, Kaneko K. Validation study of the Forgotten Joint Score-12 as a universal patient-reported outcome measure. European journal of orthopaedic surgery \& traumatologie. 2015;25(7):1141-5.

55. Baumann F, Ernstberger T, Loibl M, Zeman F, Nerlich M, Tibesku C. Validation of the German Forgotten Joint Score (G-FJS) according to the COSMIN checklist: does a reduction in joint awareness indicate clinical improvement after arthroplasty of the knee? Arch Orthop Trauma Surg. 2016;136(2):257-64.

56. Sansone V, Fennema P, Applefield RC, Marchina S, Ronco R, Pascale W, Pascale V. Translation, cross-cultural adaptation, and validation of the Italian language Forgotten Joint Score-12 (FJS-12) as an outcome measure for total knee arthroplasty in an Italian population. BMC Musculoskelet Disord. 2020;21(1):23.

57. Singh V, Yeroushalmi D, Lygrisse KA, Simcox T, Long WJ, Schwarzkopf R. The influence of obesity on achievement of a 'forgotten joint' following total knee arthroplasty. Arch Orthop Trauma Surg. 2022;142(3):491-9.

58 World_Health_Organization. The Asia Pacific perspective: Redefining obesity and its treatment. 2000

59. Goyal T, Sethy SS, Paul S, Choudhury AK, Das SL. Good validity and reliability of forgotten joint score-12 in total knee arthroplasty in Hindi language for Indian population. Knee Surg Sports Traumatol Arthrosc. 2021;29(4):1150-6.

60. Sabah SA, Alvand A, Beard DJ, Price AJ. Evidence for the validity of a patient-based instrument for assessment of outcome after revision knee arthroplasty. The Bone \& Joint Journal. 2021;103-B(4):627-34.

61. HD F, GJ M, MD J, SAHR W, HC R, G K. Responsiveness and ceiling effects of the Forgotten Joint Score-12 following total hip arthroplasty. Bone Joint Res. 2016;5(3):87-91.

62. Aday LA, Cornelius LJ. Designing and conducting health surveys: a comprehensive guide. New Jersey: John Wiley and Sons; 2006.

63. Bernard HR. Research methods in anthropology: Qualitative and quantitative approaches. Maryland: Rowman and Littlefield; 2017.

64. Groves RM. Theories and methods of telephone surveys. Ann Rev Sociol. 1990;16(1):221-40.

65. Shadid MB, Vinken NS, Marting LN, Wolterbeek N. The Dutch version of the Forgotten Joint Score: test-retesting reliability and validation. Acta Orthop Belg. 2016;82(1):112-8.

66. Hau MYT, Menon DK, Chan RJN, Chung KY, Chau WW, Ho KW. Twodimensional/three-dimensional EOS $^{\mathrm{TM}}$ imaging is reliable and comparable to traditional X-ray imaging assessment of knee osteoarthritis aiding surgical management. Knee. 2020;27(3):970-9.

67. Chien CW, Wang JD, Yao G, Hsueh IP, Hsieh CL. Agreement between the WHOQOL-BREF Chinese and Taiwanese versions in the elderly. J Formos Med Assoc. 2009;108(2):164-9.

\section{Publisher's Note}

Springer Nature remains neutral with regard to jurisdictional claims in published maps and institutional affiliations.

Ready to submit your research? Choose BMC and benefit from:

- fast, convenient online submission

- thorough peer review by experienced researchers in your field

- rapid publication on acceptance

- support for research data, including large and complex data types

- gold Open Access which fosters wider collaboration and increased citations

- maximum visibility for your research: over $100 \mathrm{M}$ website views per year

At BMC, research is always in progress.

Learn more biomedcentral.com/submissions 